\title{
Compensation for Interaction Torques During Single- and Multijoint Limb Movement
}

\author{
PAUL L. GRIBBLE AND DAVID J. OSTRY \\ McGill University, Montreal, Quebec H3A 1B1, Canada
}

Gribble, Paul L. and David J. Ostry. Compensation for interaction torques during single- and multijoint limb movement. J. Neurophysiol. 82: 2310-2326, 1999. During multijoint limb movements such as reaching, rotational forces arise at one joint due to the motions of limb segments about other joints. We report the results of three experiments in which we assessed the extent to which control signals to muscles are adjusted to counteract these "interaction torques." Human subjects performed single- and multijoint pointing movements involving shoulder and elbow motion, and movement parameters related to the magnitude and direction of interaction torques were manipulated systematically. We examined electromyographic (EMG) activity of shoulder and elbow muscles and, specifically, the relationship between EMG activity and joint interaction torque. A first set of experiments examined single-joint movements. During both singlejoint elbow (experiment 1) and shoulder (experiment 2) movements, phasic EMG activity was observed in muscles spanning the stationary joint (shoulder muscles in experiment 1 and elbow muscles in experiment 2). This muscle activity preceded movement and varied in amplitude with the magnitude of upcoming interaction torque (the load resulting from motion of the nonstationary limb segment). In a third experiment, subjects performed multijoint movements involving simultaneous motion at the shoulder and elbow. Movement amplitude and velocity at one joint were held constant, while the direction of movement about the other joint was varied. When the direction of elbow motion was varied (flexion vs. extension) and shoulder kinematics were held constant, EMG activity in shoulder muscles varied depending on the direction of elbow motion (and hence the sign of the interaction torque arising at the shoulder). Similarly, EMG activity in elbow muscles varied depending on the direction of shoulder motion for movements in which elbow kinematics were held constant. The results from all three experiments support the idea that central control signals to muscles are adjusted, in a predictive manner, to compensate for interaction torques-loads arising at one joint that depend on motion about other joints.

\section{N T R O D U C T I O N}

Although much has been learned about the control of limb movements at single joints, the way in which the nervous system coordinates neural commands to muscles for multijoint movements (such as reaching) is less well understood. Results from studies of single-joint movement cannot be extrapolated easily to multijoint situations because during the latter, the control problem is complicated by the presence of intersegmental dynamics or "interaction torques"-rotational forces that arise at one joint (e.g., the shoulder) because of motion of limb segments about other joints (e.g., motion of the lower arm about the elbow). In typical single-joint experiments in which

\footnotetext{
The costs of publication of this article were defrayed in part by the payment of page charges. The article must therefore be hereby marked "advertisement" in accordance with 18 U.S.C. Section 1734 solely to indicate this fact.
}

all joints but one are fixed in place these interaction torques, although present, do not affect motion. However, during multijoint movement, or when more than one joint is free to move, interaction torques arise that are dependent in a nonlinear fashion on motion at adjacent joints (see Hollerbach and Flash 1982 and Eqs. 3 and 4 in this paper). In the present paper, we report studies of single- and multijoint arm movement in which electromyographic measures are used to assess the extent to which the nervous system adjusts control signals to muscles to compensate for interaction torques.

Numerous studies of multijoint limb movement have been reported that demonstrate invariance of movement kinematics, suggesting that interaction torques are in some manner dealt with. For example, subjects produce straight hand paths for planar movements in different directions and at different speeds (Morasso 1981), despite large changes in both the magnitude and direction of interaction torques (Hollerbach and Flash 1982). Similar results have been reported for other studies of planar movement (Haggard et al., 1995; Soechting and Lacquaniti 1981), and three-dimensional movements (Morasso 1983). Although these findings show that movement kinematics can be preserved in the face of interaction torques (Hollerbach and Flash 1982), the mechanisms by which this occurs cannot be determined on the basis of kinematics alone. For example, it has been demonstrated that forces resulting from muscle mechanics and reflexes may, at least for some movements, counteract the effects of limb dynamics (Gribble et al., 1998). Indeed, a central notion of equilibrium-point control models is the idea that muscle properties dominate loads arising from limb dynamics and that simple control signals that take no account of interaction torques may underlie movement. Thus kinematic invariance alone could arise either from explicit compensation for interaction torques in control signals or because of the effects of muscle mechanical properties and reflexes. Without direct measurements of muscle activity it is not possible to distinguish between these possibilities.

Recent reports have addressed the issue of compensation for interaction torques during multijoint arm movement. In one study, it was demonstrated that during a step tracking task involving simultaneous rotation at the elbow and wrist, electromyographic (EMG) activity in wrist muscles varied with interaction torques introduced by motion of the lower arm (Cooke and Virji-Babul 1995; also see Virji-Babul and Cooke 1995). Koshland, Gerilovsky, and Hasan (1991) report a similar dependence of wrist muscle activity on elbow movement even under conditions in which the wrist was immobilized. In another study, it was shown that although normal subjects were able to accurately reproduce a pantomimed "slicing" gesture 
involving shoulder and elbow rotation, patients without proprioception made systematic movement errors consistent with a failure to offset joint interaction forces at the elbow due to motion of the upper arm (Sainburg et al. 1993; also see Ghez and Sainburg 1995). In a related study, Sainburg, Ghilardi, Poizner, and Ghez (1995) demonstrated that when elbow motion was held constant and the amplitude of shoulder movement was varied (thus affecting the interaction torque arising at the elbow), changes in the timing of elbow muscle activity were observed that varied systematically with changes in interaction torques. The authors conclude that this reflects adjustments in control signals to offset the effects of limb dynamics.

The possibility that even in single-joint movements, control signals are adjusted to offset the effects of interaction torques is raised by Almeida, Hong, Corcos, and Gottlieb (1995). These authors report phasic EMG activity at a stationary joint (e.g., the shoulder) that acts to stabilize the limb in the face of motion about an adjacent joint (e.g., the elbow). The authors note that the form of EMG activity at both the stationary and moving joint is similar and suggest that this may reflect a simple strategy whereby the nervous system may compensate for the effects of intersegmental dynamics.

Although the results of the studies described in the preceding text suggest that the nervous system uses compensatory adjustments in muscle activity to counteract forces arising from limb dynamics, a systematic exploration of the relation between interaction torques, movement kinematics and muscle activity patterns has not been reported. The present experiments extend previous work in a number of ways. We experimentally manipulate movement parameters (both amplitude and speed) related to the magnitude and direction of interaction torques during both single- and multijoint movements. We assess the relationship between interaction torques and EMG activity in both single- and double-joint muscles to assess the extent to which control signals compensate for interaction torques. In this way we explore how EMG activity varies in relation to interaction torques and the degree to which electromyographic changes systematically anticipate upcoming loads arising from limb dynamics.

We consider two types of movements involving shoulder and elbow motion. First, we examined single-joint movements in which subjects pointed to targets that could be achieved by rotating one joint alone (e.g., the elbow). Nevertheless, the stationary joint (e.g., the shoulder) was unrestrained and thus was affected by interaction torques arising due to motion of the other joint. Movement speed was manipulated experimentally, and hence the magnitude of the interaction torque arising at the stationary joint likewise varied (see Eqs. 3 and 4). Two experiments of this type were conducted. Experiment 1 involved single-joint elbow flexion movements, and in experiment 2, each subject performed single-joint shoulder flexion movements from the start position. We examined EMG activity in muscles spanning the stationary joint and, in particular, the relationship between the magnitude of EMG activity in muscles at that joint and the magnitude of the interaction torque arising due to motion about the nonstationary joint. The timing of EMG activity relative to movement onset also was examined.

In a third experiment, subjects performed movements to targets that involved simultaneous rotation at both the shoulder and elbow. The direction of movement at one joint was varied systematically (flexion or extension), whereas the kinematics of the other joint were held constant. The effect of this manipulation was to vary the sign of the interaction torque arising at the joint at which kinematics were constant. The relationship between the magnitude of the interaction torque and EMG activity was examined.

In both kinds of experiments, anticipatory changes in EMG activity were observed that varied in proportion to interaction torques arising during movement. These results suggest that control signals to muscles are adjusted in a predictive manner to compensate for forces arising from multijoint dynamics.

\section{METHODS}

\section{Subjects}

Ten subjects ( 7 male, 3 female) between the ages of 25 and 51 participated in experiment 1 . Nine of these subjects also were tested in experiments 2 and 3. The experiments were conducted on separate days. Subjects reported no history of neurological or musculo-skeletal disorders. The experimental procedures used in these studies have been approved by the Ethics Committee of the Department of Psychology, McGill University.

\section{Apparatus}

Figure $1 A$ illustrates the experimental setup. Subjects were seated in front of a table with a glass surface, with their right arm abducted at the shoulder and supported by two custom-made air sleds-one located under the upper arm and a second under the wrist, both secured to the limb using Velcro straps. The sleds were attached to a 50-psi compressed air source, which supported the arm against gravity and provided essentially frictionless motion of the arm in a horizontal plane containing the shoulder. The wrist was stabilized using an elastic brace. Subjects were seated with the trunk in contact with the rounded cut-out section of the tabletop (see Fig. 1). Markers were placed bilaterally on the acromion, and trunk position was monitored continuously throughout the experiment using an overhead video camera to ensure that subjects' position remained constant relative to the table.

\section{Movement tasks}

Subjects performed point-to-point movements to targets located on the glass surface. Movement speed was controlled by instructing subjects to pace each movement to auditory tones presented over a loudspeaker. Subjects were instructed to move quickly and accurately, without making corrective movements, from a single start position to a specified target. In all three experiments, both the upper and lower arm were free to move, and subjects had full vision of their limbs. Shoulder angles were defined relative to the frontal plane, such that increasing values correspond to greater amounts of shoulder extension ( $180^{\circ}$ corresponds to an upper arm position parallel to the frontal plane). Elbow joint angles were defined relative to the upper arm such that $180^{\circ}$ corresponds to full extension of the lower arm.

In experiment 1 (see Fig. $1 B$ ), three targets were used. The target positions were determined separately for each subject, and involved 20,40 , and $60^{\circ}$ of elbow flexion from the start position, and no shoulder movement. The initial position of the limb was the same for all movements (shoulder angle was $130^{\circ}$ and elbow angle was $120^{\circ}$ ). Subjects were told that the movements could be achieved by only moving their forearm, although nothing prevented subjects from moving their upper arm. For each target, three movement speeds were tested (see RESULTS). For each of the nine experimental conditions ( 3 amplitudes $\times 3$ speeds), 20 movements were recorded. Rest periods 


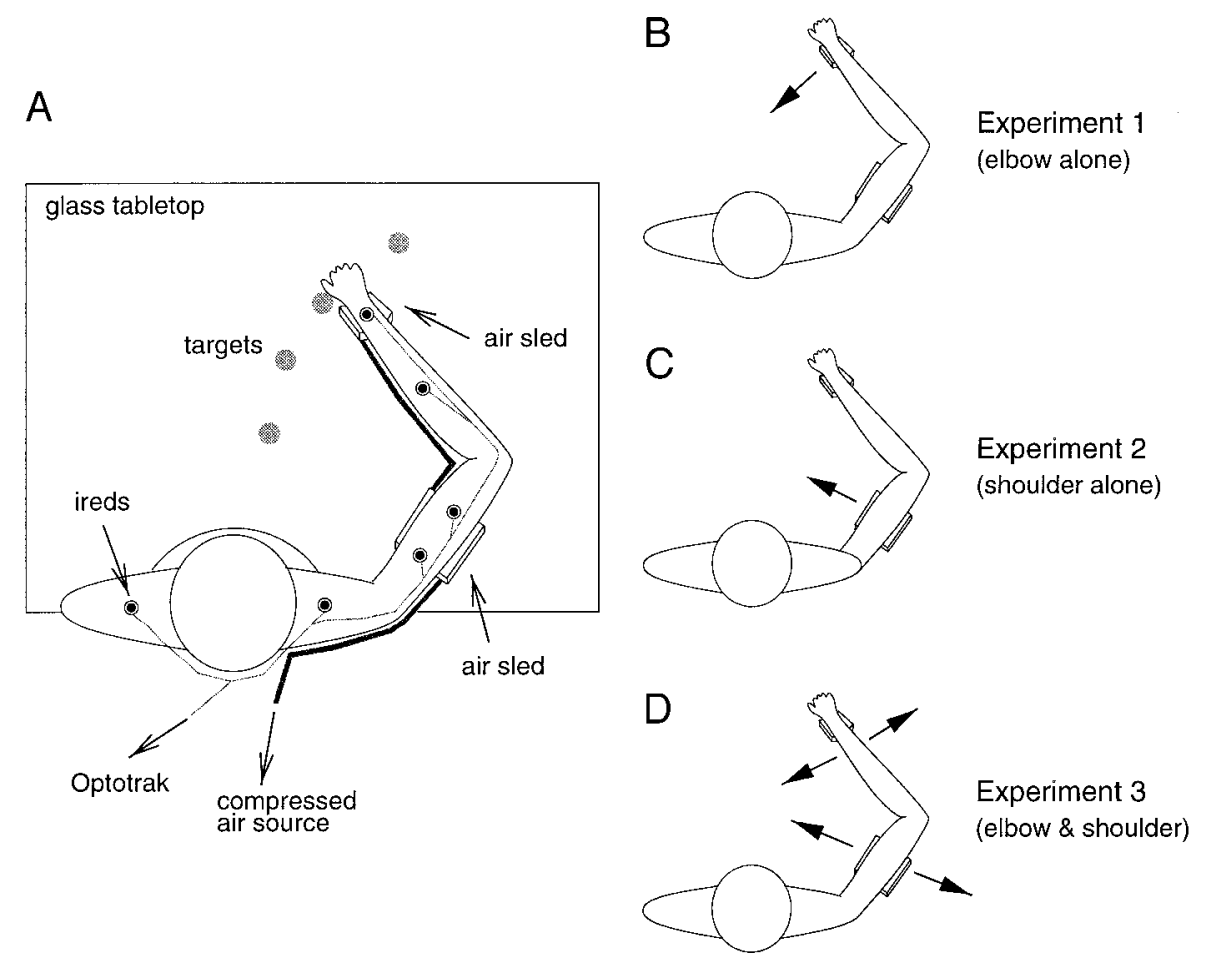

FIG. 1. Apparatus and experimental design. A: a compressed-air system supported the arm against gravity and allowed for frictionless motion in a horizontal plane to targets located on a glass surface. Six infrared emitting diodes (IREDs) located on the trunk, upper arm, and lower arm were used to compute shoulder and elbow angles during movement. Electromyographic (EMG) signals were recorded using bipolar surface electrodes from posterior deltoid, clavicular head of pectoralis, biceps long head, triceps lateral head, brachioradialis, biceps short head, and triceps long head. $B-D$ : movement tasks. $B$ : experiment 1 involved single-joint elbow flexions. Dependence of shoulder muscle EMG activity on elbow movement velocity was assessed. $C$ : in experiment 2 subjects performed single-joint shoulder flexions, and the dependence of elbow muscle EMG activity on shoulder movement velocity was assessed. $D$ : subjects in experiment 3 performed multijoint movements involving simultaneous shoulder and elbow motion. Movement kinematics at 1 joint were held constant and the direction of motion about the other joint was varied. were included to reduce subject fatigue. In all three experiments the order of the experimental conditions was randomized across subjects.

In experiment 2 (see Fig. $1 C$ ), each subject made movements from the same initial position $\left(170^{\circ}\right.$ at the shoulder and $100^{\circ}$ at the elbow) to three targets. The targets involved 20,40 , and $60^{\circ}$ of shoulder flexion from the start position, and no elbow rotation (no movement of the lower arm relative to the upper arm). Subjects were told that they could achieve the targets using shoulder rotation only, but as in experiment 1, their forearm nevertheless was free to move. Three movement speeds were tested for each target (see RESULTS). Twenty movements were recorded for each of the nine experimental conditions.

In experiment 3 (see Fig. 1D), subjects performed multijoint movements involving simultaneous flexion or extension at both the shoulder and elbow. Four targets were placed on the glass surface representing $20^{\circ}$ shoulder flexion with $30^{\circ}$ elbow flexion, $20^{\circ}$ shoulder flexion with $30^{\circ}$ elbow extension, $20^{\circ}$ shoulder extension with $30^{\circ}$ elbow flexion, and $20^{\circ}$ shoulder extension with $30^{\circ}$ elbow extension. The initial limb position was the same for all targets $\left(130^{\circ}\right.$ at the shoulder and $110^{\circ}$ at the elbow). Two movement speeds were tested for each target (see RESULTS). In each of the eight experimental conditions (4 targets $\times 2$ speeds), 20 movements were recorded.

\section{Signal recording}

Time-varying position of the limb was recorded using Optotrak (Northern Digital), an optoelectronic position sensing device that tracks the three-dimensional positions of infra-red emitting diodes (IREDs). Six IREDs were attached to the torso, upper arm, and lower arm and were used to compute shoulder and elbow joint angles (see Fig. 1A). Movement signals were sampled at $200 \mathrm{~Hz}$ and digitally low-pass filtered at $8 \mathrm{~Hz}$ off-line using a second-order Butterworth filter implemented in Matlab (Mathworks).

EMG activity of seven limb muscles associated with shoulder and elbow movement was recorded using bipolar surface electrodes (Neuromuscular Research Center). Each electrode consists of two $1 \times 10$ $\mathrm{mm}$ parallel silver bars placed $10 \mathrm{~mm}$ apart. Electrodes are housed in a compact case containing a $10 \times$ preamplifier. Recordings were made from posterior deltoid (a shoulder extensor), clavicular head of pectoralis (shoulder flexor), biceps brachii long head (2-joint flexor acting primarily at the elbow), biceps brachii short head (2-joint flexor acting at the shoulder and elbow), triceps brachii lateral head (elbow extensor), triceps brachii long head (2-joint extensor acting at the shoulder and elbow), and brachioradialis (elbow flexor). In a pilot study, activity of the sternocostal head of pectoralis also was recorded for two subjects. The patterns of results obtained were the same as those for the clavicular head. EMG signals were analogue low-pass filtered at $600 \mathrm{~Hz}$ and digitally sampled at 1,200 Hz. The resulting signals were digitally band-pass filtered between 30 and $300 \mathrm{~Hz}$ and fullwave rectified. For purposes of visualization and for scoring the onset and end of phasic EMG bursts (see following text), the rectified signals were digitally low-pass filtered at $50 \mathrm{~Hz}$.

For each muscle, electrode placement was verified by having subjects perform test maneuvers involving both free movement and isometric force adjustments. For posterior deltoid, the electrode was placed such that phasic activity was observed during shoulder extension. The electrode recording the clavicular head of pectoralis was placed so that activity was observed during shoulder flexion but not during shoulder abduction. Although both the long and short heads of biceps cross the gleno-humeral joint, the long head acts primarily as an elbow flexor (Yamaguchi et al. 1997). Thus the electrode for recording the long head of biceps was located in a position where activity was seen during elbow flexion, but no activity was observed during shoulder flexion. The electrode for recording the short head of biceps was positioned so that activity was observed for both elbow and shoulder flexion. Similarly, the electrode for recording the lateral head of triceps was in a position where activity was seen during elbow extension but not during shoulder extension, whereas for the long head of triceps, the electrode was placed so that activity was observed during both elbow and shoulder extension. For brachioradialis, a single-joint elbow flexor, the electrode was placed so that activity was seen during elbow flexion.

\section{Data analysis}

For each trial, kinematic records and EMG signals were timealigned to the start of movement, which was scored using the tangen- 
tial velocity of an IRED located on the hand. Movement start and end were scored at $5 \%$ of peak tangential velocity. Shoulder and elbow joint movement amplitudes were computed as the difference between joint angles at the start and end of movement. Average velocities of shoulder and elbow movements were computed as the ratio of movement amplitude to movement duration. In the single-joint experiments (experiments 1 and 2), we found that the average movement velocities observed in the three nominal speed conditions tended to overlap. To maximize the difference between mean movement velocities, data associated with different movement speeds were combined and then resorted into three equal sized, nonoverlapping groups according to average movement velocity-slow, medium, and fast. This procedure was carried out separately for each target condition.

Time-varying interaction torques at the shoulder and elbow were computed based on the equations of motion for a two-link planar arm (derived using Lagrangian techniques) (see Hollerbach and Flash 1982). The following equations give the equations of motion.

$$
\begin{array}{r}
N_{1}=\ddot{\theta}_{1}\left(I_{1}+I_{2}+m_{2} l_{1} l_{2} \cos \theta_{2}+\frac{m_{1} l_{1}^{2}+m_{2} l_{2}^{2}}{4}+m_{2} l_{1}^{2}\right)+\ddot{\theta}_{2}\left(I_{2}+\frac{m_{2} l_{2}^{2}}{4}\right. \\
\left.+\frac{m_{2} l_{1} l_{2}}{2} \cos \theta_{2}\right)-\frac{m_{2} l_{1} l_{2}}{2} \dot{\theta}_{2}^{2} \sin \theta_{2}-m_{2} l_{1} l_{2} \dot{\theta}_{1} \dot{\theta}_{2} \sin \theta_{2} \\
N_{2}=\ddot{\theta}_{2}\left(I_{2}+\frac{m_{2} l_{2}^{2}}{4}\right)+\ddot{\theta}_{1}\left(I_{2}+\frac{m_{2} l_{1} l_{2}}{2} \cos \theta_{2}+\frac{m_{2} l_{2}^{2}}{4}\right) \\
+\frac{m_{2} l_{1} l_{2}}{2} \dot{\theta}_{1}^{2} \sin \theta_{2}
\end{array}
$$

$N_{1}$ and $N_{2}$ are shoulder and elbow torque, $\ddot{\theta}_{1}$ and $\ddot{\theta}_{2}$ are shoulder and elbow acceleration, $\dot{\theta}_{1}$ and $\dot{\theta}_{2}$ are shoulder and elbow velocity, $\theta_{1}$ and $\theta_{2}$ are shoulder and elbow joint angles, $I_{1}$ and $I_{2}$ are the moments of inertia of the upper and lower arm about their centers of mass, $m_{1}$ and $m_{2}$ are the masses of the upper and lower arms, and $l_{1}$ and $l_{2}$ are the lengths of the upper and lower arms.

For purposes of the present paper, we define interaction torques as $T_{\mathrm{s}}$, the net torque at the shoulder that depends on motion of the lower arm, and $T_{\mathrm{e}}$, the net torque at the elbow that depends on motion of the upper arm:

$$
\begin{gathered}
T_{\mathrm{s}}=\ddot{\theta}_{2}\left(I_{2}+\frac{m_{2} l_{2}^{2}}{4}+\frac{m_{2} l_{1} l_{2}}{2} \cos \theta_{2}\right) \\
-\frac{m_{2} l_{1} l_{2}}{2} \dot{\theta}_{2}^{2} \sin \theta_{2}-m_{2} l_{1} l_{2} \dot{\theta}_{1} \dot{\theta}_{2} \sin \theta_{2} \\
T_{\mathrm{e}}=\ddot{\theta}_{1}\left(I_{2}+\frac{m_{2} l_{1} l_{2}}{2} \cos \theta_{2}+\frac{m_{2} l_{2}^{2}}{4}\right)+\frac{m_{2} l_{1} l_{2}}{2} \dot{\theta}_{1}^{2} \sin \theta_{2}
\end{gathered}
$$

Joint accelerations and velocities were computed by numerically differentiating the position signals. Lengths $l_{1}$ and $l_{2}$ were measured for each subject ( $l_{2}$ included the length of the hand). Mass $m_{2}$ and moment of inertia $I_{2}$ were estimated in proportion to overall height and weight measurements for each subject, using anthropometric tables (Winter 1990).

For each movement trial, the onset and end of the first phasic EMG burst in each muscle was scored using an interactive computer program. On a trial-by-trial basis, a baseline measure of EMG activity was computed for each muscle as the mean activity during a 100-ms window, selected $300 \mathrm{~ms}$ before the start of movement. The onset of the first EMG burst was scored as the time at which the EMG signal rose three standard deviations above the mean baseline level and remained above that level for $\geq 50 \mathrm{~ms}$. The end of the EMG burst was scored as the time at which muscle activity returned to the baseline level and remained below baseline for $\geq 50 \mathrm{~ms}$.

For each movement trial, the area associated with the first phasic burst of EMG activity was determined for each muscle by computing the integral of the signal between the onset and end of the burst. For each subject, the total set of values obtained for each muscle was normalized to $z$ scores, which allows for comparisons across subjects. The normalization procedure was carried out separately for each experiment.

\section{RES ULTS}

We show that during single-joint elbow and shoulder movements, phasic EMG activity is observed in muscles which act about the stationary joint-activity that resembles the agonistantagonist bursts typically associated with movement. Moreover, we show that the onset of this activity precedes movement and that the magnitude of this activity varies with the speed of movement at the nonstationary joint (and hence with the magnitude of the interaction torque produced by the moving segment). This supports the idea that there is centrally specified predictive compensation for interaction torques (torques arising at the stationary joint due to motion about an adjacent joint). Data from experiment 3 also will be presented that support this idea in the context of multijoint movements.

It has been reported previously in the context of perturbation studies (Gomi and Osu 1996) and muscle cocontraction measurements (Gribble and Ostry 1998) that the activity of doublejoint arm muscles (biceps short head and triceps long head) primarily is related to elbow motion. Accordingly, for the purposes of the analyses in the present paper, we have grouped the double-joint muscles together with the single-joint elbow muscles (biceps long head, brachioradialis and triceps lateral head). Data from experiment 3 also support the idea that double-joint muscle activity is primarily related to motion of the elbow, at least for the movements tested here (see Experiment 3-multijoint movements).

\section{Experiments 1 and 2: single-joint movement}

Figure $2 A$ shows, for a single subject, mean EMG activity as a function of time during a $40^{\circ}$ single-joint elbow flexion movement (experiment 1). Means were computed over 20 individual trials. Figure $2 B$ shows EMG activity during a $40^{\circ}$ single-joint shoulder flexion movement (experiment 2 ). In both cases, typical phasic patterns of agonist and antagonist EMG activity were observed for muscles acting about the moving joint. In addition, phasic activity was seen in muscles which act about the stationary joint. For example, in Fig. $2 A$, phasic activity was observed in pectoralis and deltoid-single-joint shoulder muscles - that resembles the typical pattern of agonist and antagonist EMG bursts associated with shoulder movement, even though in this case the upper arm was stationary. Similarly, in Fig. $2 B$, phasic activity was seen in biceps long head, brachioradialis, and triceps lateral head (elbow muscles), even though there was minimal movement of the lower arm relative to the upper arm. All subjects in both experiments 1 and 2 showed similar patterns.

In both single-joint elbow movements and single-joint shoulder movements, the onset of the phasic activity in flexor (agonist) muscles associated with the stationary joint preceded motion of the limb. In Fig. 3, we show histograms that depict the distribution of onset times of the first EMG burst in each muscle, relative to movement onset (pooled over all subjects and all movement trials). Movement onset is denoted by the vertical line at $t=0$. Negative values indicate that the onset of EMG preceded movement, and positive values indicate the 
A
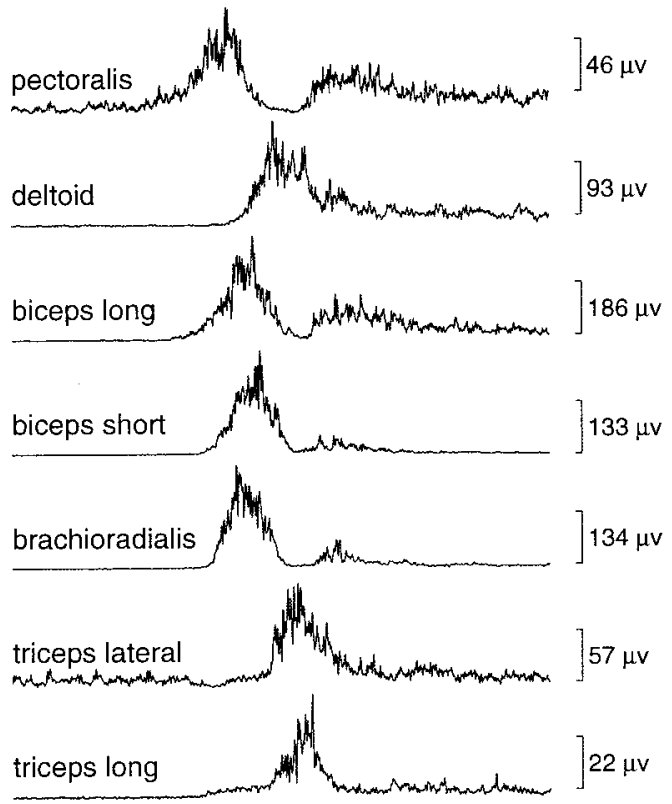

shoulder

$21 \mathrm{deg}$

elbow

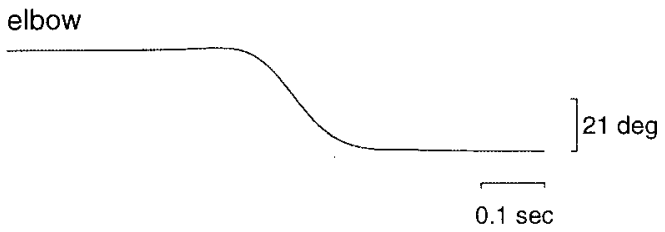

B

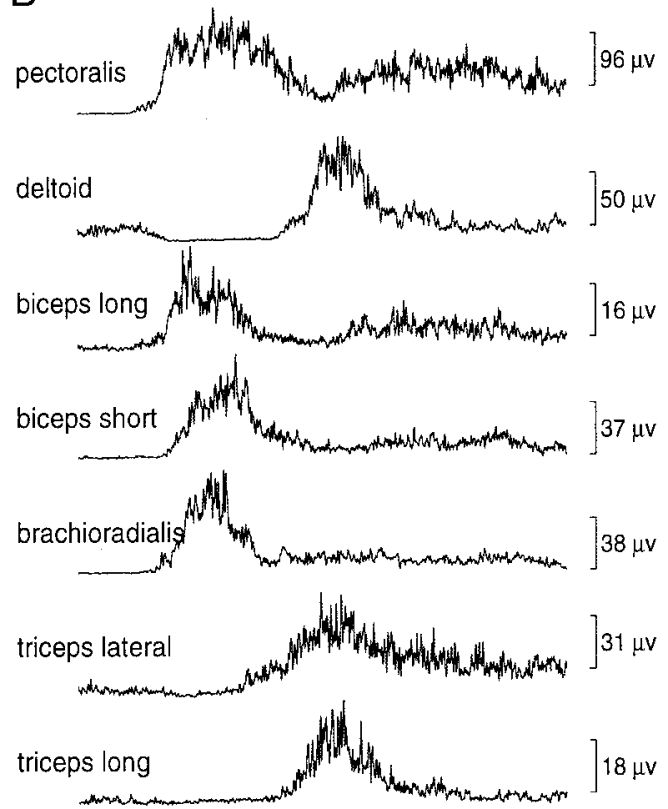

shoulder

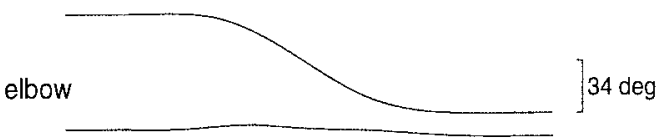

FIG. 2. Kinematic and EMG records for single-joint movements. Data shown in each panel are for 1 subject. $A$ : single-joint elbow movements. Phasic EMG activity can be seen in single-joint shoulder muscles (pectoralis and deltoid) even though there is minimal shoulder movement. Note that the onset of the pectoralis burst precedes elbow movement. $B$ : single-joint shoulder movements. Phasic EMG activity is seen in single-joint elbow and double-joint muscles even though there is minimal elbow motion. Moreover, activity in elbow (flexor) muscles precedes shoulder movement.

EMG onset occurred after movement start. The distributions of onset times for flexor muscles (which occurred before movement) are shown in the left-hand column, and the onset times for the extensor muscles are shown in the right.

Statistical tests ( $t$-tests) were carried out to assess differences in EMG onset. Data from all subjects were taken together. For single-joint elbow flexion movements (Fig. $3 A$ ), the onset of the first EMG burst in pectoralis occurred well before movement onset (mean $=-78 \mathrm{~ms}, P<0.01$ ). Similarly, Fig. $3 B$ shows that for single-joint shoulder flexion movements, the onset of phasic activity in elbow flexor muscles also occurred before movement onset $($ mean $=-39$ and $-16 \mathrm{~ms}$ for biceps long head and brachioradialis, respectively; $P<0.01$ in both cases). These data support the idea that the phasic activity (before movement) in muscles acting at the stationary joint arose centrally and was not the result of afferent inputs to motoneurons. It should be noted in addition that there was a proximal to distal temporal organization of EMG onsets in the (agonist) muscles examined here. For both elbow and shoulder movements, the onset of pectoralis activity preceded activity in elbow muscles (biceps long and short heads, and brachioradialis; $P<$
0.01 in all cases) regardless of which joint was moved (also see Experiment 3: multijoint movements).

In Fig. 4, we show patterns of mean EMG activity for single-joint movements of different speeds. Data for a single subject are shown. Thin lines show slow movements and thick lines indicate faster movements. As has been reported previously, phasic EMG activity, which varied in magnitude with movement velocity, was seen in both agonist and antagonist muscles associated with the moving joint (Brown and Cooke 1981; Mustard and Lee 1987; Corcos et al. 1989). However, the phasic activity of muscles around the stationary joint also varied with the velocity of the moving joint. In Fig. 4A, which shows data for single-joint elbow flexion movements, it can be seen that the magnitude of the phasic activity in shoulder muscles (pectoralis and deltoid) varied with the velocity of elbow movement. As elbow velocity increased, so did the magnitude of the EMG burst in shoulder muscles, even though there was little movement of the upper arm. Similarly, it can be seen in Fig. $4 B$ for single-joint shoulder movement that the magnitude of phasic activity in the single-joint elbow muscles (biceps long head, brachioradialis and triceps lateral head) varied with the speed of shoulder movement. 


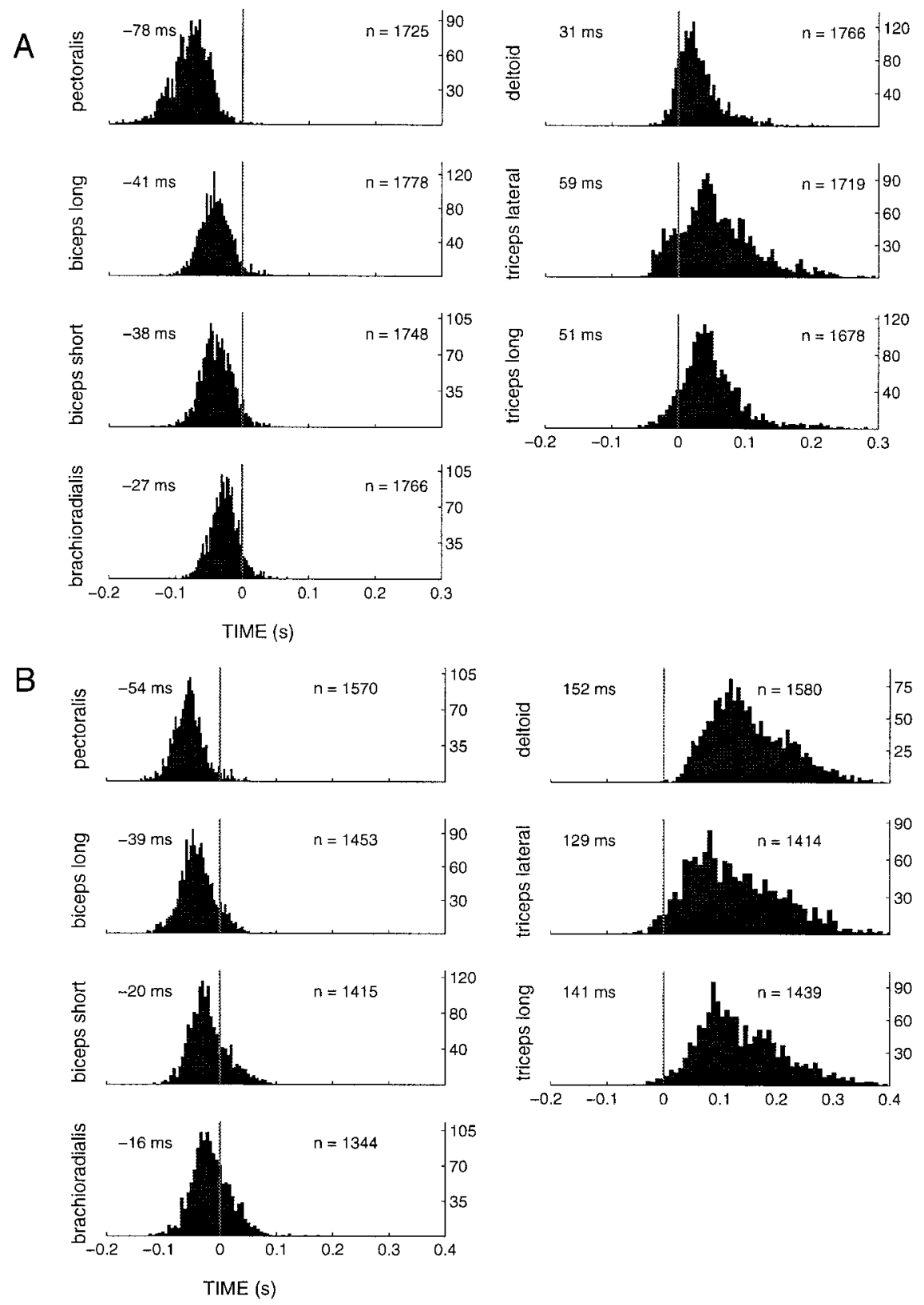

FIG. 3. Histograms showing the distribution of onset times of the 1st EMG burst in each muscle in single-joint elbow movements (experiment $1, A$ ) and single-joint shoulder movements (experiment 2, B). Movement onset is denoted by a vertical line at $t=0$. Data shown are pooled across all subjects and all experimental conditions. A: mean onset of pectoralis $(-78 \mathrm{~ms})$, a single-joint shoulder flexor, preceded elbow movement onset. $B$ : mean onset of single-joint elbow flexors (biceps long head, $-39 \mathrm{~ms}$; brachioradialis, $-16 \mathrm{~ms})$ as well as biceps short head $(-20 \mathrm{~ms})$, a double-joint flexor, preceded shoulder movement.

If a joint such as the shoulder is to remain stationary, torques at the shoulder that arise due to motion of the lower arm about the elbow, as well as torques due to elbow muscle activity, must be offset by appropriate changes in shoulder muscle activity. This pattern can be seen in Fig. 5. In Fig. 5A, timevarying joint position and velocity, interaction torques, and muscle activation patterns are shown for single-joint elbow flexion movements at three speeds. Mean data for one subject are shown; other subjects showed similar patterns. Thin lines indicate slow movements and thick lines show faster movements. Fig. $5 B$ shows comparable patterns for single-joint shoulder flexion movements. In $A$ it can be seen that a torque was produced at the shoulder (right) due to elbow motion that was first in the extension direction (positive values), then in the flexion direction (negative values). The magnitude of this torque varied with the velocity of elbow movement (see Eqs. 3 and 4). Phasic activity was observed in single-joint shoulder muscles-first in pectoralis, to offset the extension interaction torque, and then in deltoid, to oppose the flexion interaction torque. Moreover, as elbow velocity increased, the magnitude of this activity increased to offset the higher interaction torque arising at the shoulder.

Similarly, in single-joint shoulder movements (Fig. 5B), a torque was produced at the elbow (left) due to shoulder motion. As the velocity of shoulder movement increased, the magnitude of the interaction torque at the elbow increased. EMG activity in single-joint elbow muscles (biceps long head and triceps lateral head) likewise increased in proportion to the interaction torque. At the beginning of shoulder movement, phasic activity was seen in biceps long head that preceded and served to offset the effect of the interaction torque at the elbow in the extension direction. This was followed by activity in triceps lateral head that opposed the interaction torque in the flexion direction. 
A

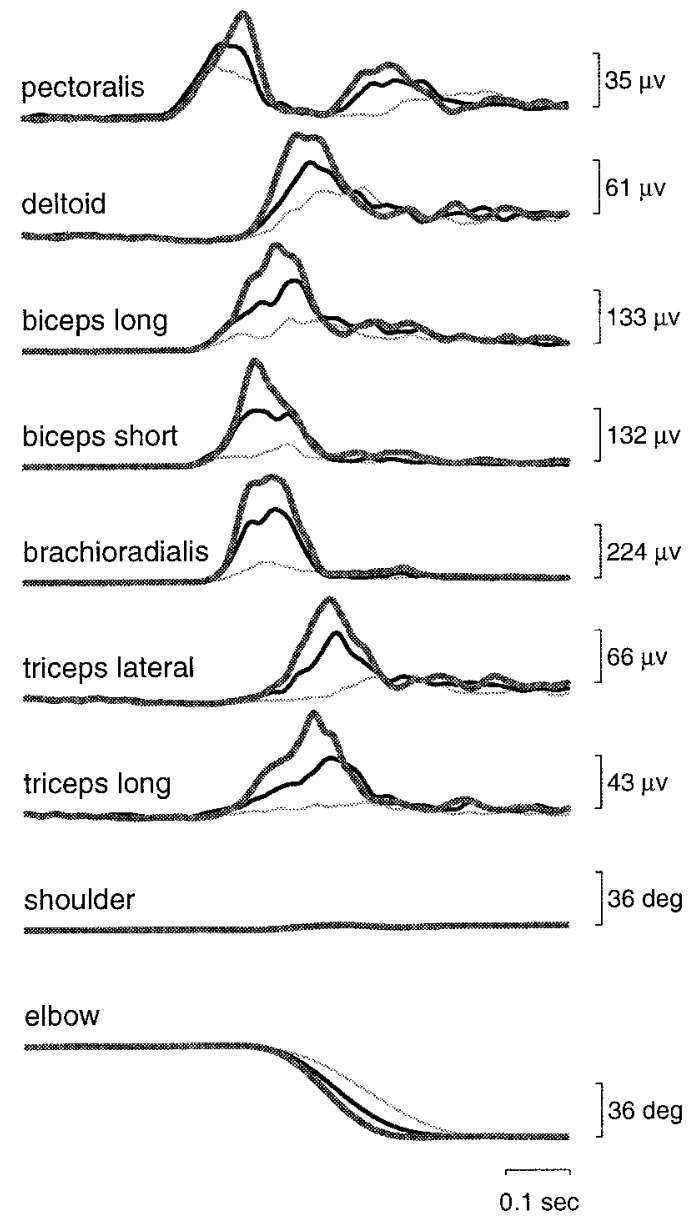

$\mathrm{B}$

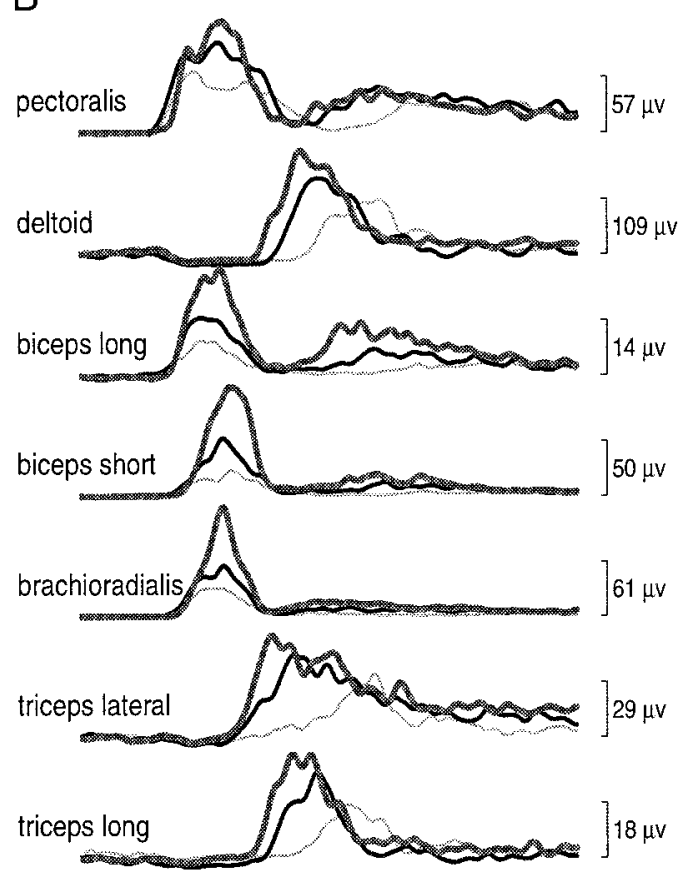

shoulder

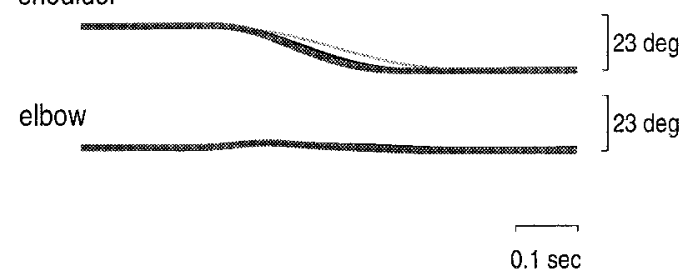

FIG. 4. Mean EMG activity and kinematic records for single-joint movements at 3 different speeds. A: magnitude of EMG activity in single-joint shoulder muscles (pectoralis and deltoid) varied with the speed of elbow movement, despite minimal shoulder motion in all cases. Data for 1 subject are shown. $B$ : single-joint elbow and double-joint EMG activity varied depending on the speed of single-joint shoulder motion. Thin lines denote slow movements, thick lines show fast movements.

Although anticipatory muscle activity at the stationary joint varied systematically with the magnitude of upcoming interaction torque, it also can be seen in both Fig. 5, $A$ and $B$ that small-amplitude changes nevertheless occurred in the position of the stationary joint (the shoulder in $A$ and the elbow in $B$ ) over the course of movement. This might indicate a lack of complete compensation for interaction torques by muscles acting at the stationary joint.

The relationship between muscle activity and movement speed is shown in Fig. 6. A shows, for experiment 1 (single-joint elbow movements), normalized area of the first EMG burst in each muscle as a function of elbow movement velocity. In each panel, three movement amplitudes are shown, from left to right, 20, 40, and $60^{\circ}$. Data were normalized and for purposes of visualization were averaged across subjects. The bottom right panel shows elbow amplitude as a function of movement velocity to indicate the kinematic patterns associated with each experimental condition. For the $20^{\circ}$ movements, average velocity ranged from 70 to $130^{\circ} \mathrm{s}$. For $40^{\circ}$ and $60^{\circ}$ movements, average velocity ranged from 130 to $200 \%$ s and 160 to $270 \%$, respectively. One-way repeated measures ANOVA was used to test whether elbow movement amplitude remained constant across the three elbow velocity conditions. Statistically significant differences were observed
$(P<0.01)$, although they were very small compared with the overall movement amplitudes $\left(0.9-1.8^{\circ}\right)$. Thus the changes observed in EMG activity may be attributable to changes in movement velocity.

For each of the three different movement amplitudes tested, one-way repeated measures ANOVA was used to assess the effect of movement speed on normalized EMG activity. For elbow and double-joint muscles (biceps long and short heads, brachioradialis, and triceps long and lateral heads), EMG activity increased as a function of elbow velocity $(P<0.01$ in all cases except for the $20^{\circ}$ target, for which only biceps long head and triceps long head showed significant differences). This dependence of agonist and antagonist EMG activity on movement speed is typical of point-to-point limb movements (Brown and Cooke 1981; Corcos et al. 1989; Mustard and Lee 1987). However, it also can be seen that single-joint shoulder EMG activity (pectoralis and deltoid) varied depending on the velocity of elbow movement $(P<0.01$ in all cases except the $20^{\circ}$ target, which showed no reliable differences). This systematic dependence of shoulder EMG activity on elbow movement was present even though there was minimal movement at the shoulder (average shoulder movement amplitude ranged from 0.5 to $3.1^{\circ}$ across subjects). 
A
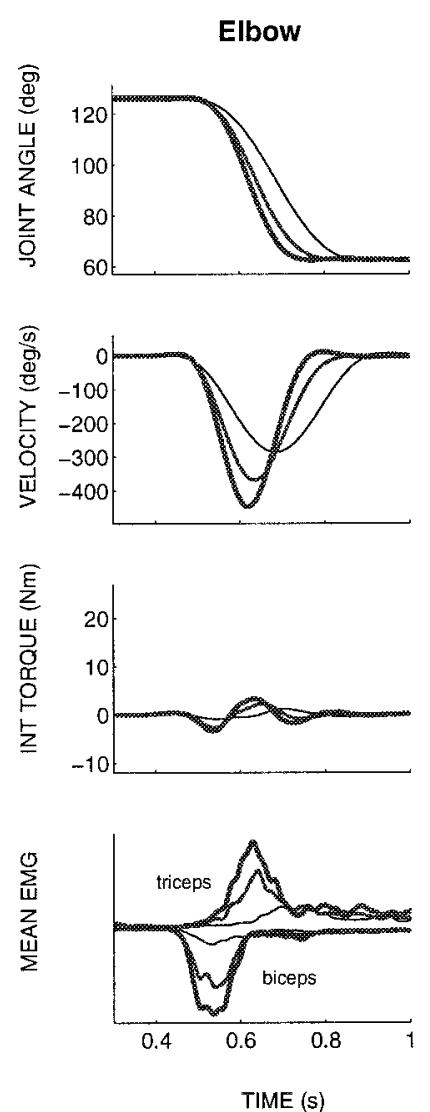

Shoulder
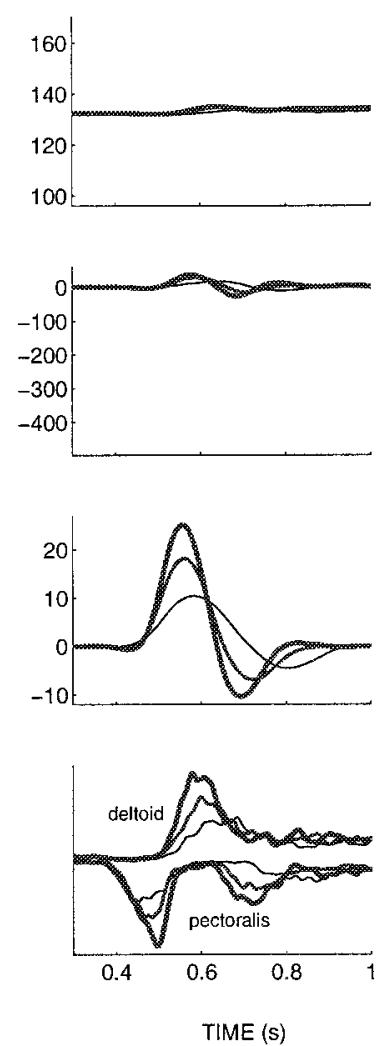

B
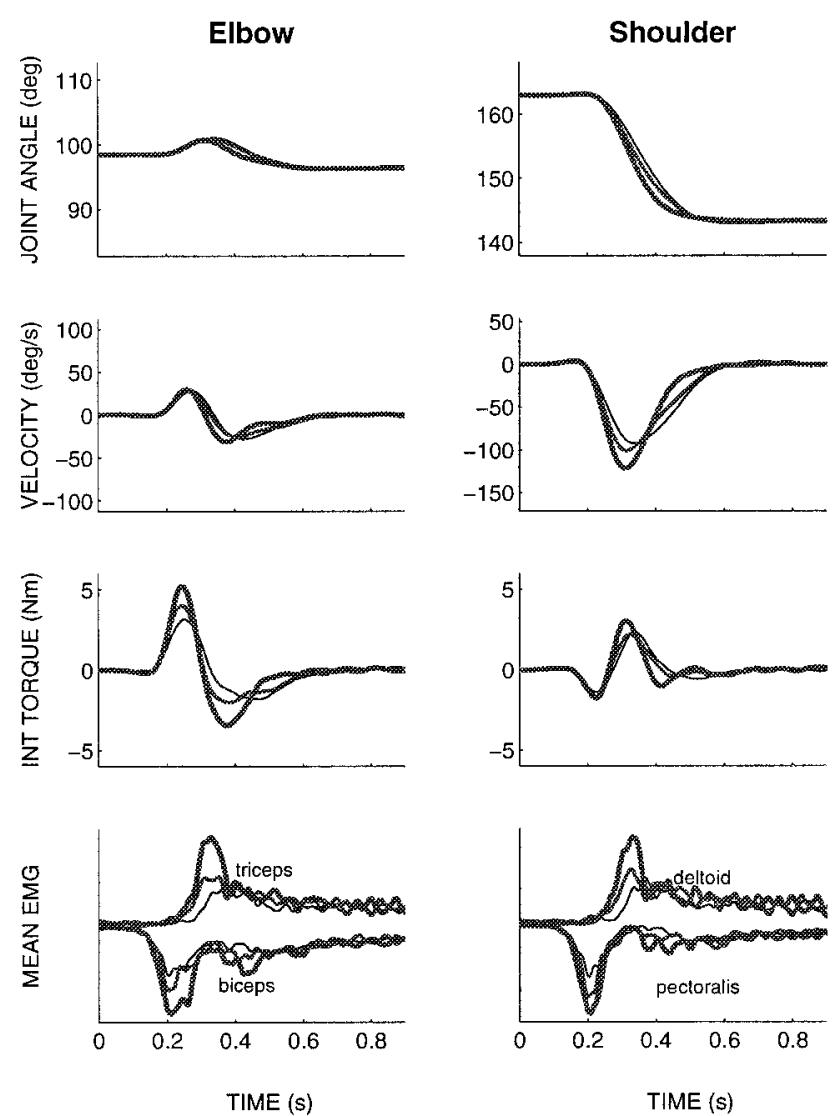
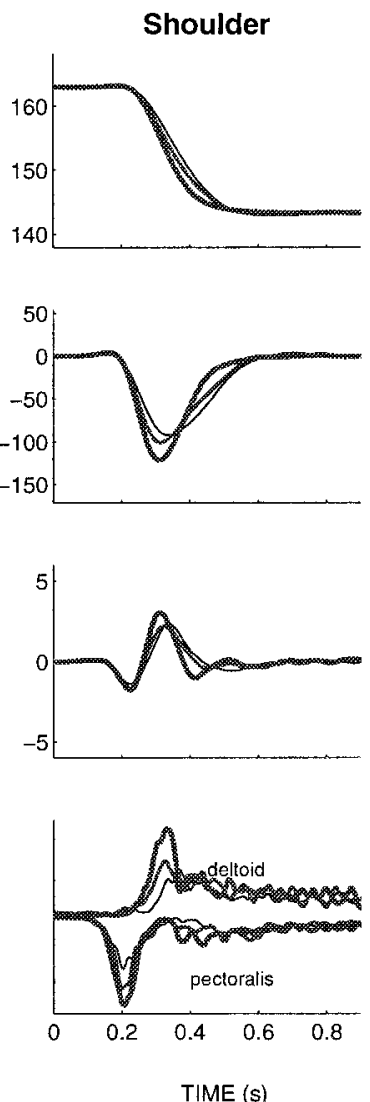

FIG. 5. Time-varying joint position, velocity, interaction torque and muscle activation patterns for single-joint elbow (experiment $1, A$ ) and shoulder (experiment 2, B) movements at 3 speeds. Data shown in each panel are for 1 subject. $A$ : increases in elbow velocity were accompanied by increases in the magnitude of the interaction torque at the shoulder (due to elbow motion) and likewise increases in the magnitude of EMG activity in single-joint shoulder muscles (pectoralis and deltoid). Shoulder motion during all 3 movements was minimal. $B$ : as the speed of shoulder movement increased, the magnitude of the interaction torque at the elbow (due to shoulder motion) increased, as did the magnitude of EMG activity in single-joint elbow muscles. Little elbow motion was observed in all cases. Thin lines show slow movements and thick lines indicate fast movements.

Although shoulder movements were small, the possibility exists that the observed changes in shoulder muscle activity were related to differences in shoulder kinematics rather than to changes in elbow movement speed. To test for this possibility, we examined, for each movement target separately, the dependence of shoulder kinematics (movement amplitude and average velocity) on elbow speed. Using repeated measures ANOVAs, we found that there were no systematic changes in either shoulder amplitude or average velocity as elbow speed varied $\left(P>0.01\right.$ for all tests except the $20^{\circ}$ target). For the $20^{\circ}$ target, statistically significant differences were observed in shoulder position $\left(<1^{\circ}\right)$ and average velocity $(<3 \%$ s); however, these changes were small relative to overall movement amplitude and velocity.

Figure $6 B$ shows the dependence of EMG activity on the velocity of shoulder movement, for single-joint shoulder flexions (experiment 2). For the purposes of visualization, data shown are averaged over subjects. Figure $6 B$ (bottom right) indicates the kinematic patterns associated with the single-joint shoulder movements tested in experiment 2 . For $20^{\circ}$ shoulder movements, average velocity ranged from 50 to $100 \%$ s. For 40 and $60^{\circ}$ movements, average velocity ranged from 60 to $130 \%$ and 90 to $180 \%$, respectively. The dependence of EMG ac- tivity on shoulder movement velocity was assessed using oneway repeated measures ANOVAs. Consistent with previous reports, single-joint shoulder muscle activity (pectoralis and deltoid) varied with shoulder movement velocity $(P<0.01)$. In addition, single-joint elbow and double-joint muscle activity (biceps long and short heads, brachioradialis, and triceps lateral and long heads) varied with the velocity of shoulder movement $(P<0.01)$ - even though minimal movement occurred at the elbow (average elbow movement amplitude for different subjects ranged from 1.8 to $5.9^{\circ}$ ). One-way repeated measures ANOVAs were used to verify, for each target separately, that shoulder movement amplitude did not vary with shoulder velocity $\left[P>0.01\right.$ for all 3 targets except the $40^{\circ}$ target, for which small $\left(<1.9^{\circ}\right)$ but significant differences were observed, $P<0.01]$. Thus the changes observed in EMG activity may be attributable to changes in movement velocity. In addition we assessed the possibility that differences in elbow EMG activity might be due to undesired changes in elbow movement amplitude or velocity for the different shoulder movement speeds that were tested. Using one-way repeated measures ANOVAs for each target condition separately, we found that neither elbow movement amplitude nor average velocity varied with shoulder velocity $(P>0.01$ for all 3 targets). 
A
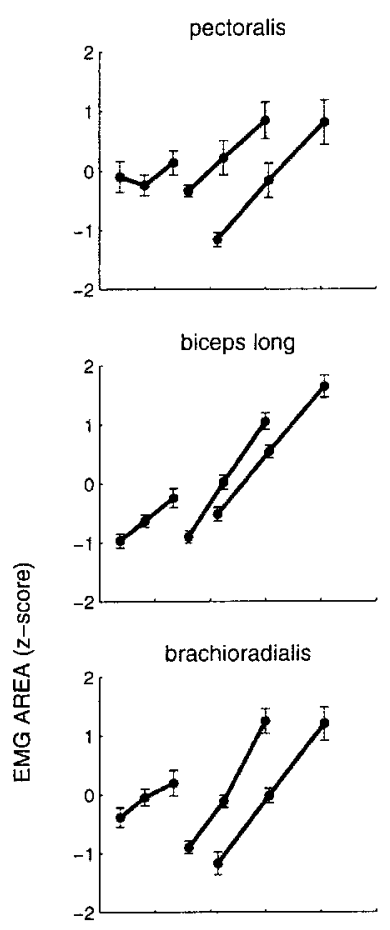

triceps long

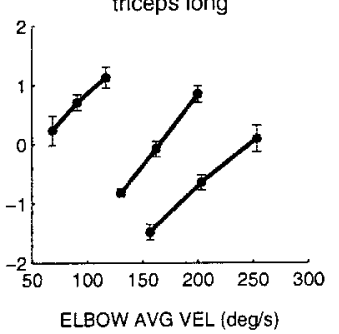

deltoid

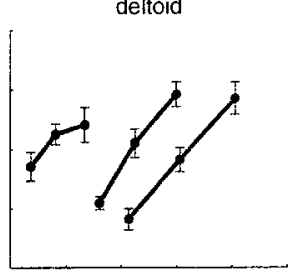

biceps short

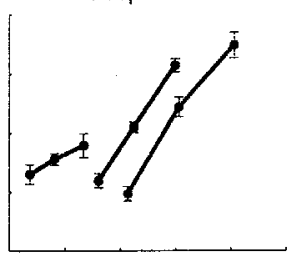

triceps lateral

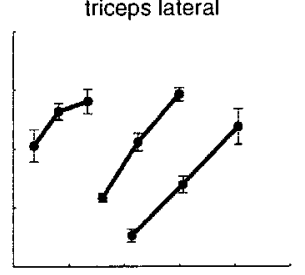

Kinematics

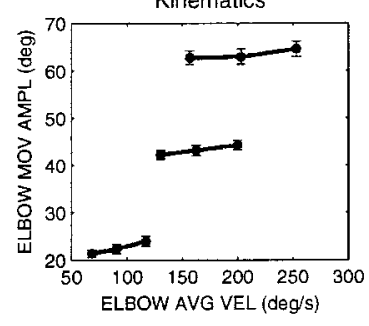

B

Single-Joint Shoulder Movements
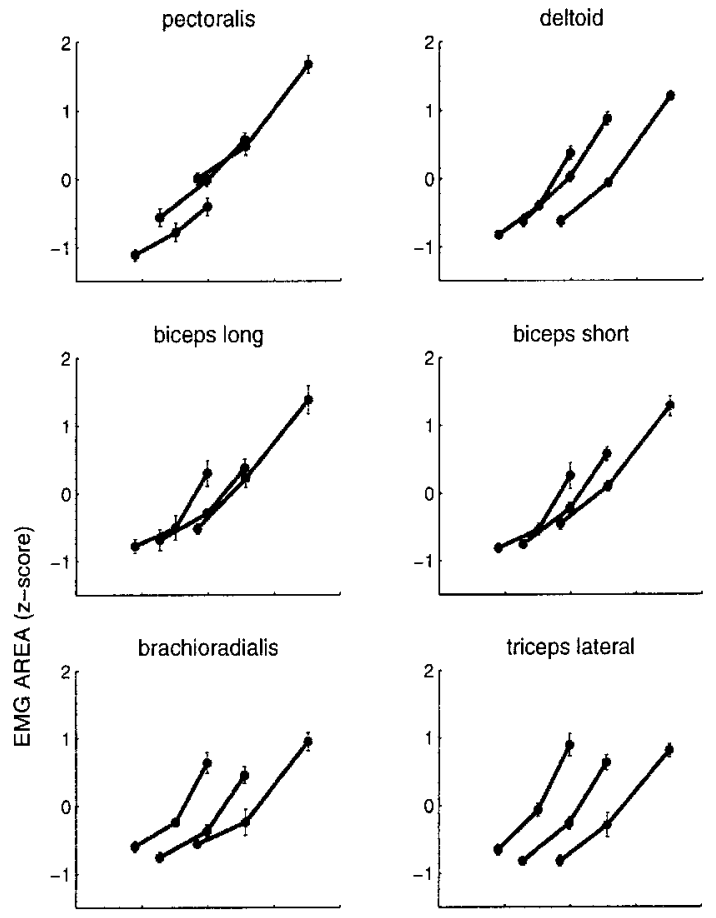

triceps lateral

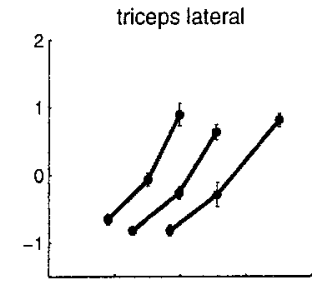

triceps long
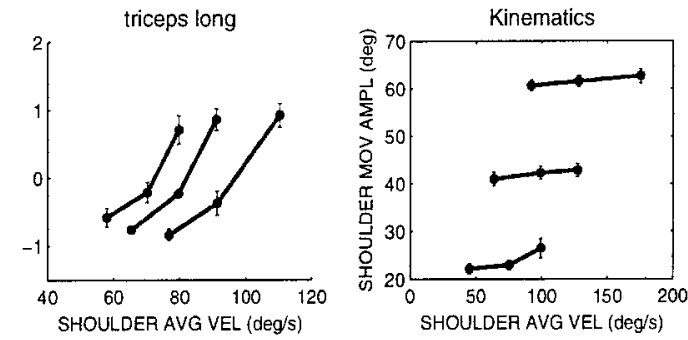

FIG. 6. Relationship between muscle activity (normalized area of the 1st EMG burst) and average movement speed in single-joint elbow movements (experiment $1, A$ ) and single-joint shoulder movements (experiment 2, B). Data are averaged across all subjects. In each panel data for 3 movement amplitudes are shown (from left to right, 20, 40, and $60^{\circ}$ ). Bottom right plot of each panel shows the kinematic patterns associated with the movements (movement amplitude is plotted against average velocity). A: elbow and double-joint muscles showed a typical dependence of burst area on elbow movement velocity. In addition, EMG activity of pectoralis and deltoid (both single-joint shoulder muscles) varied with the speed of elbow movement. $B$ : EMG activity of single-joint elbow and double-joint muscles varied with the speed of shoulder movement.

The relationship between movement velocity and interaction torque was assessed using one-way repeated measures ANOVAs for each movement target separately. In agreement with Eqs. 3 and 4, it was found that peak interaction torque at the stationary joint increased with higher movement velocities. In single-joint elbow movements, the average increase, from slow to fast movements, in peak shoulder interaction torque (torque at the shoulder due to elbow motion) was 10-20 Nm $(P<0.01$ for all 3 movement amplitudes). For single-joint shoulder movements, slightly smaller increases in elbow interaction torque were observed with increases in shoulder movement velocity (3-12 Nm, $P<0.01$ for all 3 amplitudes).

The dependence of muscle activity on interaction torque was assessed directly by examining the relationship between the maximum value of interaction torque and the integrated area under the first EMG burst for each muscle. These values are plotted in Fig. 7; mean values over all subjects and all experimental conditions are shown. In Fig. $7 A$, the relationship between peak interaction torque at the shoulder (torque arising from motion of the lower arm about the elbow) and normalized EMG activity in single-joint shoulder muscles is shown for the single-joint elbow movements in experiment 1 . For both pectoralis and deltoid, there was a significant correlation between EMG activity and peak interaction torque at the shoulder $(r=$ 0.65 for pectoralis, $r=0.54$ for deltoid; $P<0.01$ in both cases). Similarly, Fig. $7 B$ shows the relationship between normalized EMG activity in single-joint elbow muscles and double-joint muscles, and the peak interaction torque at the elbow (torque arising due to motion of the upper arm about the shoulder). In all cases, a significant correlation was observed ( $r=0.64$ for biceps long head, $r=0.68$ for biceps short head, $r=0.66$ for brachioradialis, $r=0.72$ for triceps lateral head, and $r=0.73$ for triceps long head; $P<0.01$ in all cases).

In summary, during single-joint movements at the elbow and shoulder, phasic activity was observed in single-joint muscles that act at the stationary joint. The finding that this phasic 
A

Single-Joint Elbow Movements

pectoralis

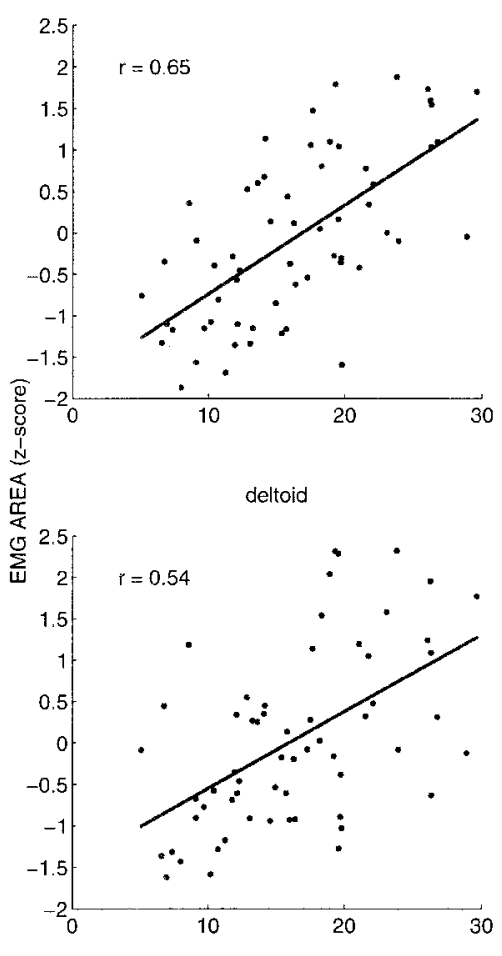

PEAK INT TORQUE $(\mathrm{Nm})$
B
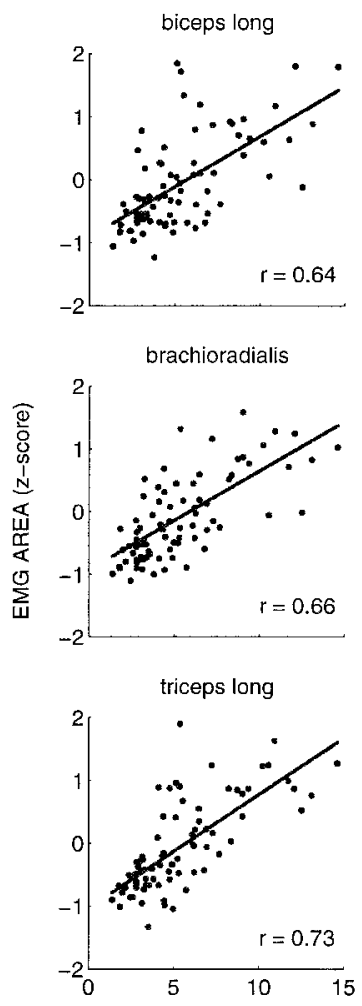

PEAK INT TORQUE (Nm)
FIG. 7. Relationship between interaction torque and normalized muscle activity. Mean data from all subjects and all experimental conditions are shown. $A$ : for single-joint elbow movements, muscle activity of pectoralis and deltoid varied as a function of peak interaction torque at the shoulder (the torque at the shoulder due to elbow motion). $B$ : for single-joint shoulder flexion movements, activity in single-joint elbow and double-joint muscles varied as a function of peak interaction torque at the elbow (the torque at the elbow due to shoulder motion). activity appeared before movement onset and the observed dependence of the amplitude of this activity on the velocity of movement at the nonstationary joint both support the idea that there are anticipatory adjustments to control signals to counteract the effects of interaction torques.

\section{Experiment 3: multijoint movements}

In this experiment, subjects performed multijoint movements involving simultaneous motion at the elbow and shoulder. Targets were placed so as to vary the relative direction of shoulder and elbow movement, thus affecting the sign of interaction torques. The data are grouped in two different ways for analysis. First, movements are considered in which the shoulder kinematics were held constant (same direction, amplitude and velocity) but the direction of elbow movement was varied (flexion or extension). The effect of this was to vary the direction of the interaction torque at the shoulder (the torque at the shoulder arising due to elbow motion). We also consider movements in which elbow kinematics were held constant, and the direction of shoulder rotation was varied. In both cases we assessed the dependence of EMG activity in muscles acting about the joint at which kinematics were held constant, on the direction of movement at the other joint. For example, we assessed the dependence of shoulder muscle EMG on the direction of elbow rotation, for movements in which the shoulder movement direction, amplitude, and velocity were the same but the elbow movement involved either a flexion or an extension.

Figure 8 summarizes the findings of experiment 3 . We show muscle activity patterns for those muscles that act about the joint at which kinematics were held constant. In Fig. 8A, we show the dependence of EMG activity in single-joint shoulder muscles on the direction of elbow movement for two movements in which the shoulder kinematics were held constant. Mean data for one subject are shown. In one movement, the shoulder and elbow rotated in the same direction (both flexion, thin lines), and in the other movement, they rotated in opposite directions (shoulder flexion, elbow extension-shown using thick lines). Note that the shoulder joint kinematics are essentially the same for both movements-only the direction of elbow rotation was different. Nevertheless, it can be seen that the magnitude of EMG activity in pectoralis and deltoid (single-joint shoulder muscles) varied with the direction of elbow rotation. Muscle activity was greater when the joints rotated in the same direction compared with when the shoulder and elbow rotated in opposite directions.

Similarly, Fig. $8 B$ shows the dependence of EMG activity in single-joint elbow and double-joint muscles on the direction of shoulder movement when elbow kinematics were held constant. One movement involved flexion at both joints, and the other required shoulder extension and elbow flexion. Note again that in this case the kinematics for the elbow were essentially the same, and only the direction of shoulder rotation was different. EMG activity in elbow and double-joint muscles varied depending on the direction of shoulder movement. When the joints rotated in the same direction (thin lines), muscle activity was higher than when they rotated in opposite directions (thick lines). The same pattern was observed for all subjects.

As noted earlier, there have been a number of recent reports 

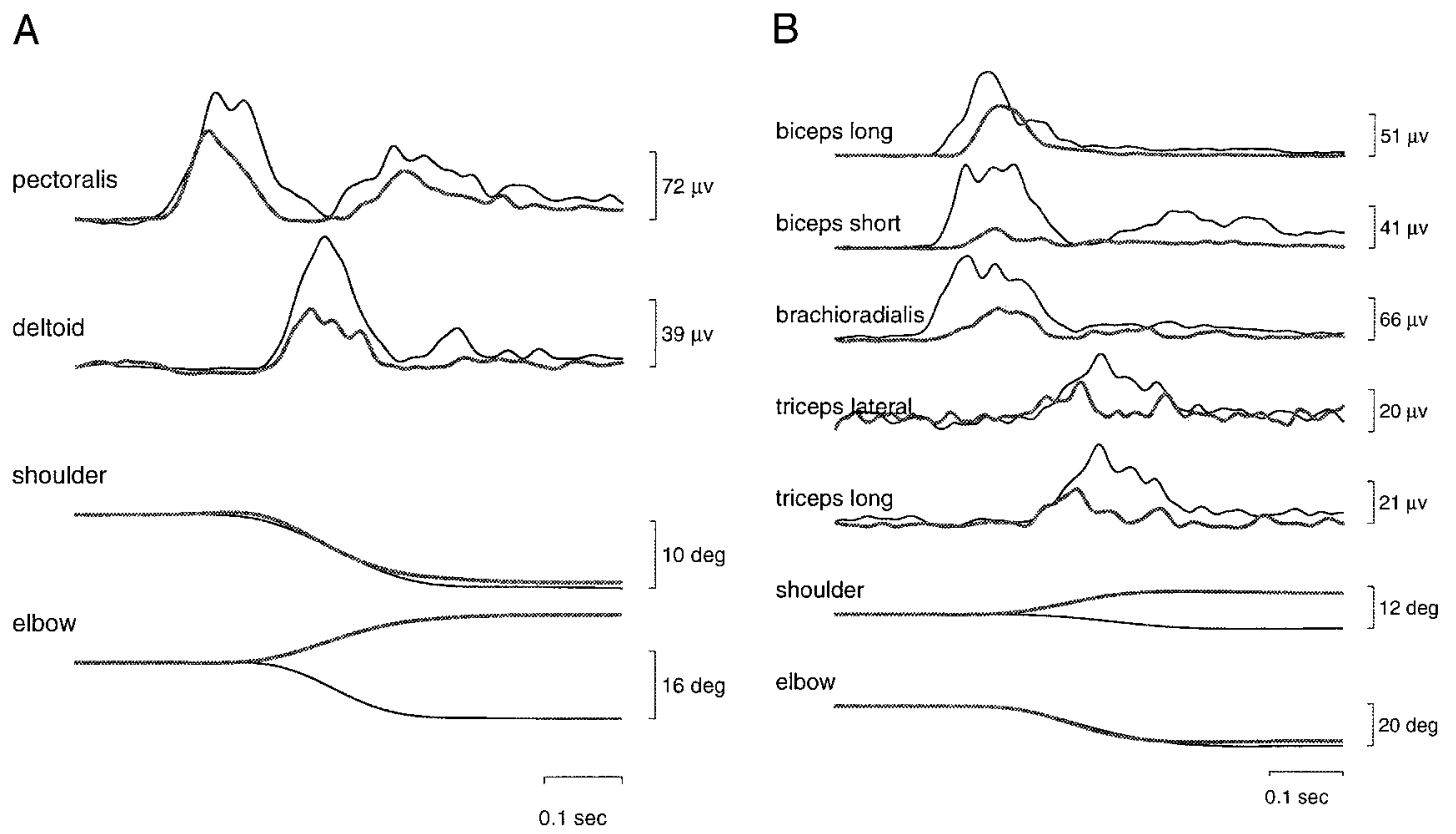

FIG. 8. Mean muscle activity and kinematic patterns for multijoint movements involving simultaneous shoulder and elbow motion (experiment 3). Data from 1 subject are shown. A: EMG activity in pectoralis and deltoid varied depending on the direction of elbow motion despite similar shoulder kinematics. Muscle activity was greater for movements in which the shoulder and elbow rotated in the same direction (and interaction torques at the shoulder opposed shoulder movement). $B$ : single-joint elbow (biceps long head, brachioradialis, and triceps lateral head) and double-joint (biceps short head and triceps long head) EMG activity associated with the same elbow motion varied depending on the direction of shoulder movement. When the elbow and shoulder rotated in the same direction, interaction torque arising at the elbow due to shoulder motion opposed the elbow movement and greater activity in elbow and double-joint muscles was observed. Thin lines denote movements in which the joints rotated in the same direction, and thick lines show movements in which the joints rotated in opposite directions.

suggesting that double-joint muscle activity is related primarily to motion about the elbow joint. Data from the present study further support this. It can be seen in Fig. $8 B$ that when the direction of shoulder movement was reversed (but the elbow kinematics were held constant), the EMG activity patterns for the double-joint muscles (biceps short head and triceps long head) in both cases displayed patterns similar to those of the single-joint elbow muscles. If the double-joint muscle activity was related primarily to shoulder movement, one would have expected to see a reversal in the order of onset of biceps short head and triceps long head activity, when the shoulder movement direction was reversed. That is, when the shoulder movement involved an extension, the triceps long head would have acted as an agonist and its activity would have preceded that of biceps short head, which would have acted as an antagonist. In contrast, it can be seen in Fig. $8 B$ that for both shoulder flexion and extension movements, biceps short head activity preceded activity in triceps long head. These data provide support for the idea that (at least for the movements tested here), double-joint muscle activity is primarily related to elbow motion.

As in experiments 1 and 2, a proximal to distal temporal organization was observed for the onset of EMG bursts in shoulder and elbow muscles-the onset of shoulder agonist muscles preceded the onset of elbow agonist activity. Statistical tests ( $t$-tests) were carried out on data pooled across subjects to assess differences in mean onset times. For movements involving shoulder and elbow flexion, mean onset of pectoralis activity $(-75 \mathrm{~ms})$ preceded mean onset of biceps long $(-52$ $\mathrm{ms})$ and short $(-58 \mathrm{~ms})$ head, as well as brachioradialis $(-36$ ms) $(P<0.01$ in all cases $)$. Likewise, for shoulder flexion/ elbow extension movements, mean onset of pectoralis activity
$(-72 \mathrm{~ms})$ preceded the onset of triceps lateral $(-31 \mathrm{~ms})$ and long $(-29 \mathrm{~ms})$ heads $(P<0.01$ in both cases). For shoulder extension/elbow flexion movements, mean onset of deltoid activity $(-62 \mathrm{~ms})$ preceded activity of biceps long $(-28 \mathrm{~ms})$ and short $(-23 \mathrm{~ms})$ head, as well as brachioradialis $(-21 \mathrm{~ms})$ $(P<0.01$ in all cases). Finally, for movements involving shoulder and elbow extension, mean onset of deltoid activity $(-57 \mathrm{~ms})$ preceded the onset of activity in triceps lateral and long heads $(-28$ and $-25 \mathrm{~ms}$, respectively; $P<0.01$ in both cases).

The data from experiment 3 are described further in Fig. 9. Time-varying position, velocity, and interaction torque at the shoulder and elbow are shown along with EMG activity in single-joint elbow and shoulder muscles. In Fig. 9A, two movements are shown - in both movements, the shoulder kinematics were held constant (flexion) but the elbow rotated either into flexion in one movement or extension in the other. It can be seen that the interaction torque arising at the shoulder due to elbow rotation (right) varied depending on the direction of elbow movement. When both joints rotated into flexion (shown using thin lines), a large interaction torque occurred at the shoulder that initially opposed shoulder flexion (positive values of torque). Conversely, when the joints rotated in opposite directions (thick lines), the interaction torque at the shoulder was opposite in sign (negative torque values) - in this case the interaction torque initially assisted the shoulder flexion movement. When muscle activation patterns are examined (bottom), it can be seen that the magnitude of single-joint shoulder (pectoralis and deltoid) EMG activity varied depending on the sign of the interaction torque at the shoulder. When the interaction torque initially opposed shoulder movement, muscle 
A
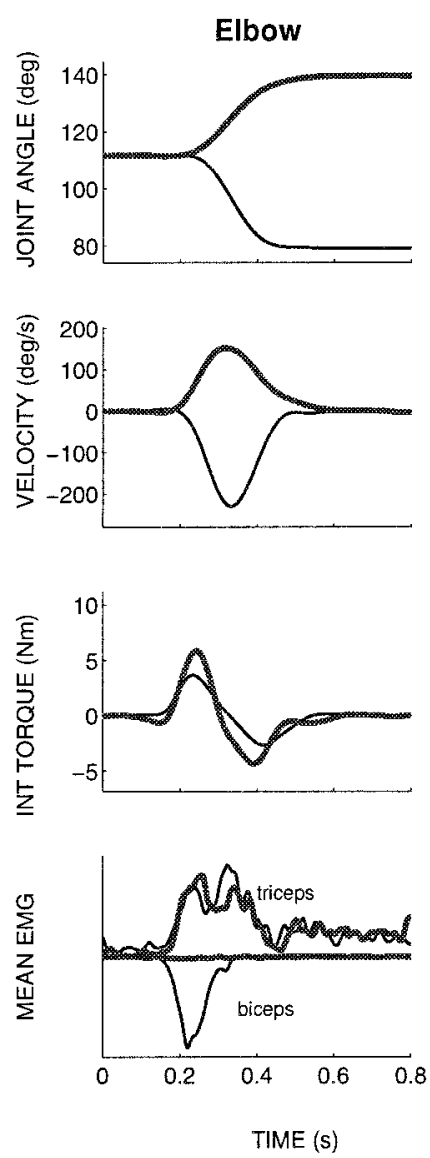

B
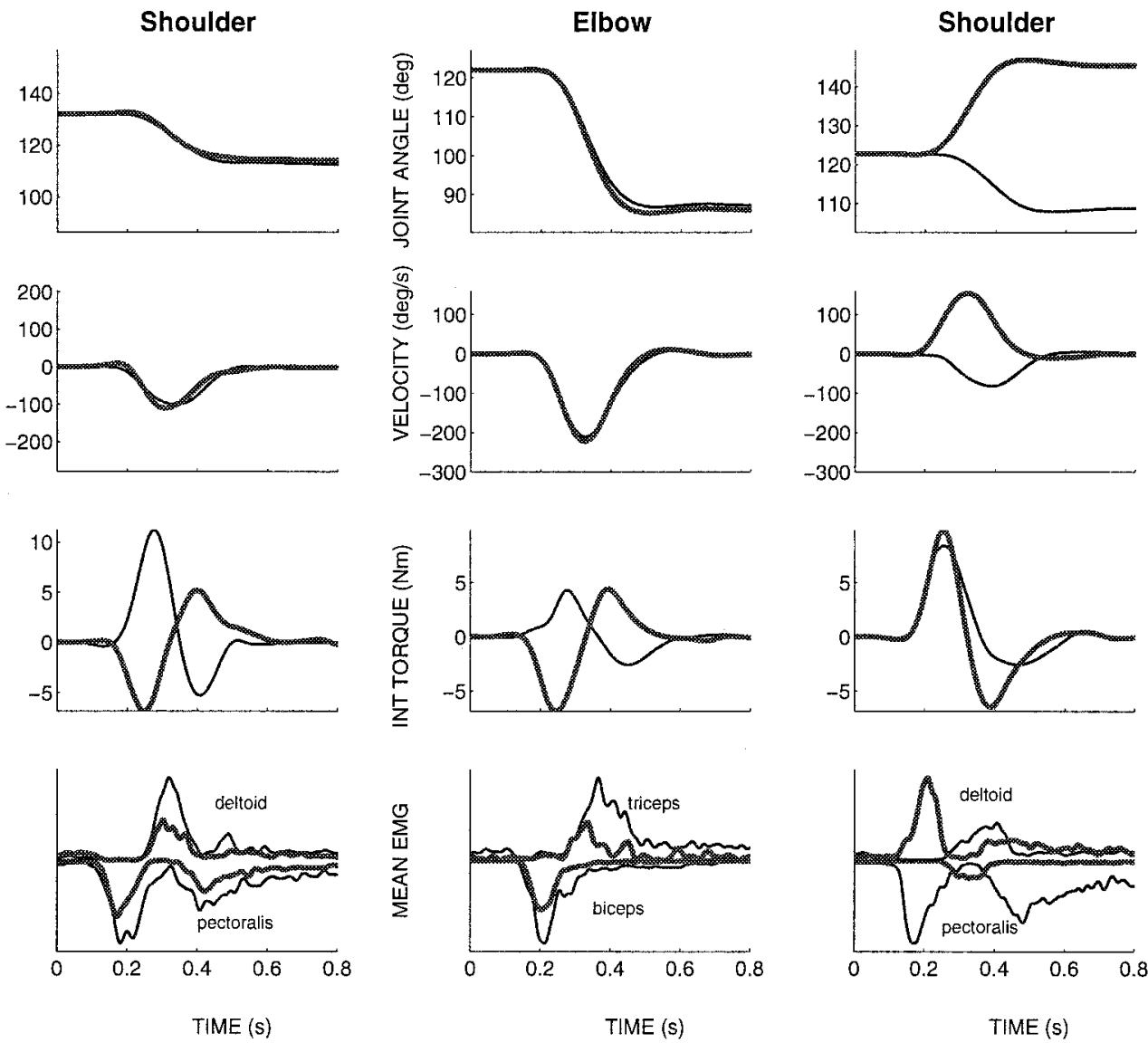

FIG. 9. Time-varying joint position, velocity, interaction torque, and muscle activity for multijoint movements in experiment 3 . Data for 1 subject are shown. A: mean data for 2 movements in which the shoulder movement kinematics were held constant (flexion), but elbow movement involved flexion in 1 case and extension in the other. When the shoulder and elbow rotated in the same direction (thin lines), an interaction torque arose at the shoulder due to elbow motion that initially opposed shoulder flexion (positive values of torque). Muscle activity in single-joint shoulder muscles (pectoralis and deltoid) was greater for these movements than for those in which the joints rotated in opposite directions (thick lines), and the interaction torque at the shoulder initially assisted shoulder movement (negative torque). $B$ : similar pattern was observed for 2 movements in which the elbow movement was held constant but the direction of shoulder rotation was varied. When movements involved both shoulder and elbow flexion, an interaction torque arose at the elbow due to shoulder motion that initially opposed elbow movement (positive values of torque). Muscle activity in single-joint elbow muscles (biceps long head and triceps lateral head) was greater than during movements in which the joints rotated in opposite directions, and the interaction torque at the elbow initially assisted elbow flexion (negative values of torque).

activity was greater than when the interaction torque assisted shoulder flexion. In the latter case, the interaction torque assisting shoulder flexion was presumably not of sufficient magnitude to produce the shoulder movement on its own, and so in this case muscle activity (e.g., pectoralis) still was required to generate sufficient net flexion torque.

Figure $9 B$ shows the same signals plotted for two movements in which the elbow kinematics were held constant, but the shoulder movement involved a flexion in one case and an extension in the other. In this case, the interaction torque arising at the elbow (left) varied depending on the direction of shoulder motion-when the joints rotated in the same direction, the elbow interaction torque initially opposed the elbow movement (positive values of torque), and elbow EMG activity increased. When the joints rotated in the opposite direction, the interaction torque at the elbow assisted the elbow flexion (negative torque values), and muscle activity in single-joint elbow muscles (biceps long head and triceps lateral head) was less. The same patterns were observed for the double-joint muscles.

The relationship between muscle activity and the relative direction of shoulder and elbow motion is shown in Figs. 10 and 11. All subjects showed similar patterns, thus the data shown are averaged across subjects. Figure 10 shows normalized EMG activity in single-joint shoulder muscles as a function of the direction of elbow motion for movements in which shoulder kinematics were held constant $\left(20^{\circ}\right.$ flexion or extension). Figure 10, bottom, indicates the kinematic parameters of the movements. Shoulder amplitude is plotted against elbow amplitude for the four targets (positive values indicate extension, negative values denote flexion). Data for two movement speeds are shown-slower movements (-, average speed $44 \%$ for shoulder and $78 \%$ s for elbow), and faster movements (- - , average speed $61 \%$ s for shoulder and $113 \%$ for elbow). It can be seen that the magnitude of EMG activity in shoulder agonist muscles (pectoralis for shoulder flexion, left half of top 

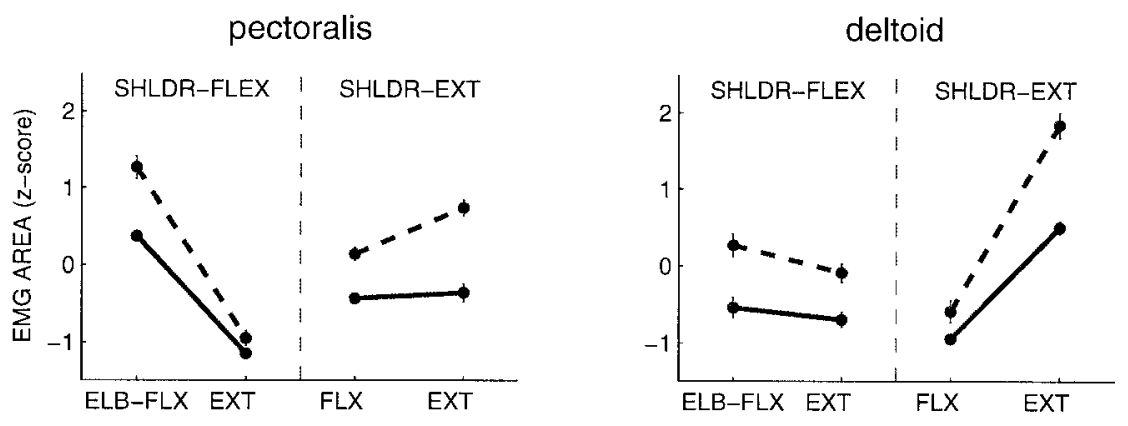

Kinematics

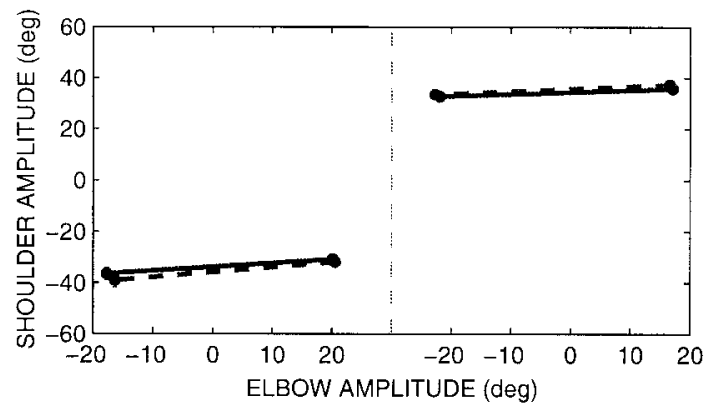

FIG. 10. Relationship between normalized EMG activity of single-joint shoulder muscles, and the direction of elbow motion, for movements in which the shoulder kinematics were held constant. Means across all subjects are shown, for movements at both slow (-) and fast (- - ) speeds. Bottom: kinematic patterns associated with each experimental condition (elbow movement amplitude is plotted against shoulder amplitude; negative values indicate flexion, positive values denote extension). For agonist muscles (pectoralis for shoulder flexion movements and deltoid for extension movements) EMG activity was greater for movements in which the shoulder and elbow rotated in the same direction and lower when they rotated in opposite directions. This pattern was less pronounced for antagonist muscles (pectoralis for shoulder extension movements and deltoid for flexion movements). left panel, and deltoid for shoulder extension, right half of right-hand panel), varied depending on the direction of elbow movement. These differences were tested using separate oneway repeated measures ANOVAs for each muscle and each movement speed condition $(P<0.01$ for both muscles, at both speeds). When the joints rotated in the same direction, muscle activity was greater than when they rotated in opposite directions. Although smaller differences were observed, a similar pattern was seen in the antagonist muscles (deltoid for shoulder flexion, left half of right-hand panel, and pectoralis for shoulder extension, right half of left-hand panel)-EMG activity was higher when the shoulder and elbow rotated in the same direction $(P<0.01$ in all cases).

The possibility exists that the changes in shoulder muscle activity shown in Fig. 10 were related to differences in shoulder kinematics rather than to changes in the direction of elbow movement. We used one-way repeated measures ANOVAs to assess this possibility for each target and movement speed separately. For the movements involving shoulder flexion, there was no change in shoulder movement amplitude or average velocity associated with different elbow movement directions (flexion vs. extension) $(P>0.01$ in all cases). For movements involving shoulder extension, small differences in shoulder amplitude and velocity were observed $(P<0.01$ for both speeds for amplitude and average velocity). The average change in shoulder movement amplitude ranged from 3 to $5^{\circ}$ across subjects, and the average change in shoulder velocity was in all cases $<10 \%$ of peak velocity. Given that these differences are small relative to the overall movements, and in light of the finding that for shoulder flexion, changes in shoulder EMG were observed even though no reliable differences in shoulder kinematics were observed, it is unlikely that the differences in shoulder EMG for shoulder extension movements were due to the changes detected in shoulder kinematics.

In Fig. 11, the relationship between elbow and double-joint muscle activity and the direction of shoulder movement is shown for movements in which elbow kinematics were held constant $\left(40^{\circ}\right.$ flexion or extension). Two movements speeds are shown-slow (-) and fast (- - -). The kinematic parameters of the movement conditions are indicated in the bottom right panel, which shows elbow movement amplitude plotted against shoulder amplitude (negative values indicate flexion, positive values denote extension). For agonist muscles (biceps long and short heads, and brachioradialis for elbow flexions, left half of left-hand panels, and triceps long and lateral heads for extensions, right half of right-hand panels), differences in EMG activity were observed for movements in which the direction of shoulder movement was reversed. When the shoulder rotated in the same direction as the elbow, EMG activity was greater than when the joints rotated in opposite directions. One-way repeated measures ANOVAs were used to test these differences for each movement speed and muscle separately $(P<0.01$ in all cases $)$. Similar, although smaller differences were seen in antagonist muscles (biceps long and short heads and brachioradialis for elbow extensions, right half of left-hand panels, and triceps long and lateral heads for elbow flexions, left half of right-hand panels $)(P<0.01$ in all cases except brachioradialis, for which $P<0.05$ for slow elbow extension movements, and for which no difference was detected in fast elbow extension movements). Again, EMG magnitudes were greater for movements in which the joints rotated in the same direction.

As in the preceding text, one-way repeated measures ANOVAs were carried out to rule out the possibility that the observed differences in elbow and double-joint muscle activity might be due to differences in elbow kinematics associated with changing the direction of shoulder movement. No significant changes in elbow amplitude or velocity were observed for different shoulder movement directions (flexion vs. extension; $P>0.01$ in all conditions, for both amplitude and velocity).

\section{I S C U S S I O N}

To assess the extent to which intersegmental dynamics are represented in control signals, we systematically manipulated 
biceps long

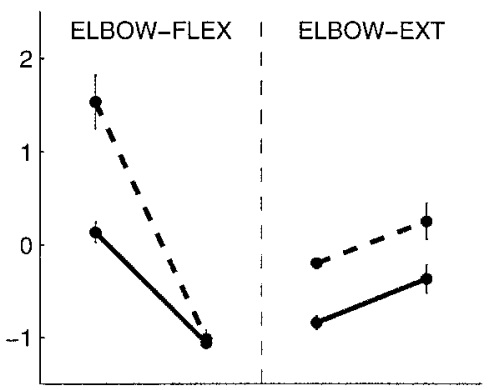

brachioradialis

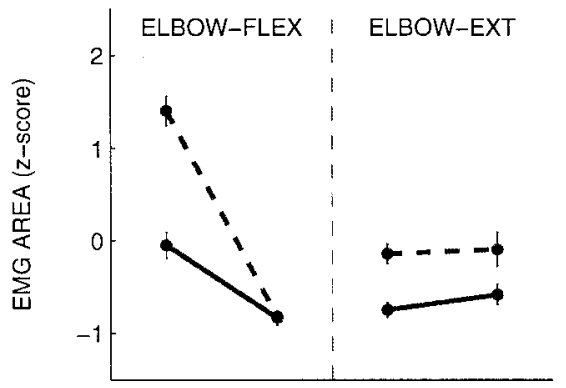

biceps short

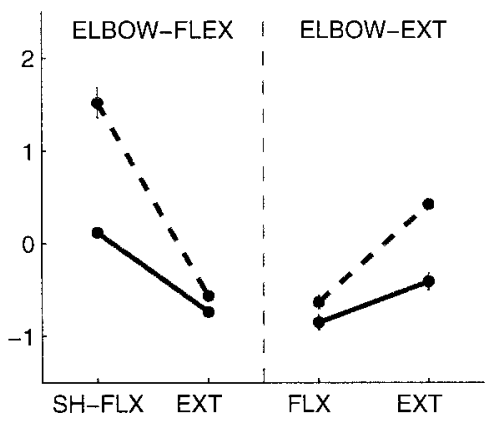

SHOULDER MOVEMENT DIRECTION triceps lateral

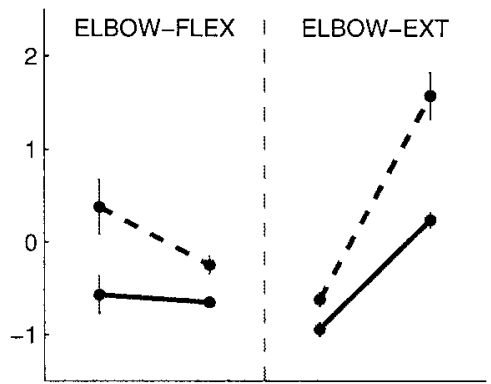

triceps long

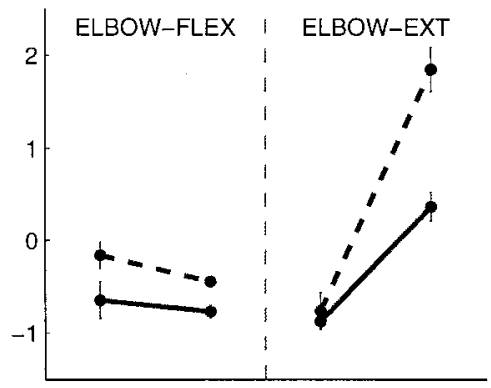

Kinematics

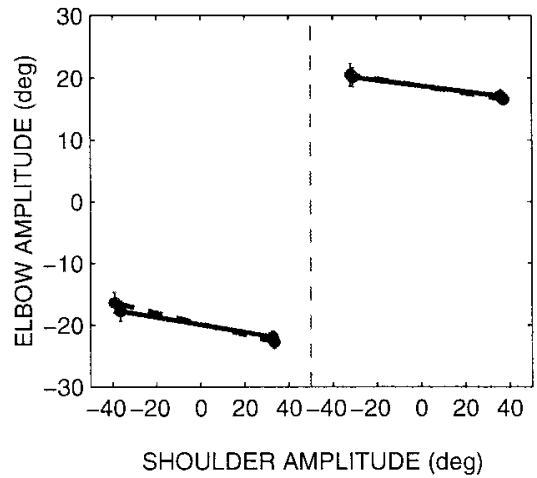

FIG. 11. Relationship between normalized EMG activity of elbow and double-joint muscles, and the direction of shoulder motion, for movements in which the elbow kinematics were held constant. Mean data across subjects are shown, for both slow (-) and fast (- - ) movements. Bottom right: elbow movement amplitude is plotted against shoulder amplitude (negative values indicate flexion, positive values denote extension). For movement agonists (biceps long and short head, and brachioradialis for elbow flexion, and triceps long and lateral heads for elbow extension), EMG activity was greater for movements in which the shoulder rotated in the same direction as the elbow and less for movements in which the joints rotated in opposite directions. A less pronounced pattern was seen for antagonist muscles (biceps long and short heads, and brachioradialis for elbow extensions, and triceps long and lateral heads for elbow flexions). parameters related to the magnitude and direction of joint interaction torques that accompany pointing movements involving the shoulder and elbow. We examined EMG activity of shoulder and elbow muscles, and specifically, the relationship between EMG activity and interaction torque. During singlejoint movements in which there was motion at only one joint, phasic EMG activity was observed in single-joint muscles spanning the stationary joint. This muscle activity preceded movement and varied in magnitude with upcoming interaction torque at that joint resulting from motion of the nonstationary limb segment. This pattern was observed both for single-joint elbow movements (movements in which the upper arm was stationary and the lower arm rotated about the elbow) and single-joint shoulder movements (movements in which the lower arm was stationary relative to the upper arm, which rotated about the shoulder).

A similar relationship between EMG activity and interaction torque was observed during multijoint movements involving simultaneous motion at the shoulder and elbow. Subjects performed movements in which movement ampli- tude and velocity at one joint was held constant while the direction of movement about a second joint was varied. When the direction of movement at the elbow was varied (flexion vs. extension) and shoulder movement kinematics were held constant, the magnitude of EMG activity in single-joint shoulder muscles varied depending on the direction of elbow motion (and hence the sign of the interaction torque arising at the shoulder). Similarly, the magnitude of EMG activity in elbow muscles varied depending on the direction of shoulder motion for movements in which elbow kinematics were held constant. The patterns of variation in EMG activity for both the single- and multijoint movements reported here are consistent with the idea that central control signals to muscles are adjusted, in a predictive manner, to compensate for interaction torques-loads arising at one joint that depend on motion about other joints.

Similar examples of anticipatory adjustments to control signals have been reported for other tasks. Studies of grip force adjustments during rapid arm movements with hand-held loads have suggested that subjects adjust control signals to finger 
muscles in a predictive manner to compensate for movement induced inertial, viscous, and elastic loads on objects (e.g., Flanagan and Wing 1997). Similarly, anticipatory adjustments in the activity of various trunk and leg muscles may be observed that offset loads on the body introduced by rapid arm movements (see de Wolf et al. 1998; van der Fits and HaddersAlgra 1998 for reviews).

A main finding of the experiments reported here is that during single-joint movement, marked phasic activity was observed in muscles acting at stationary joints. Related findings have been reported by Almeida, Hong, Corcos, and Gottlieb (1995) (also see Gottlieb et al. 1996). In their study, subjects produced single-joint elbow or shoulder flexions over three distances in a sagittal plane. EMG patterns in flexor and extensor muscles at the "focal" (moving) and "nonfocal" (stationary) joints were recorded for both movements in which the nonfocal joint was physically restrained or free to move. The authors show that for movements in which the nonfocal joint was unconstrained, phasic EMG patterns arose in muscles acting at the nonfocal joint that were similar in form to those seen in muscles at the focal joint and acted to oppose torques arising from motion at the focal joint. The authors conclude that some compensation for interaction torques occurred at the nonfocal joint. The results of the present study extend this work by demonstrating that phasic activity in muscles at the nonfocal joint arises in both single- and double-joint muscles and that the onset of this activity precedes movement. In addition, by experimentally manipulating both movement amplitude and speed, we show that the magnitude of EMG bursts in muscles at the nonfocal joint is related directly to the magnitude of upcoming interaction torques.

The finding of phasic activity in muscles acting at a stationary joint that precedes movement and varies in amplitude with upcoming interaction torques calls into question the validity of the concept of "single-joint" movement as it relates to neural control. Indeed, the single-joint movements reported here may be described more aptly as special cases of multijoint movement. Just as in the case of multijoint motion, control signals for single-joint motion must be coordinated appropriately to muscles at multiple joints to stabilize adjacent limb segments in the face of interaction torques. This multijoint coordination of control signals is required in all cases in which more than one limb segment is free to move, and intersegmental dynamics play a role.

The idea that control signals must be coordinated to muscles at multiple joints applies to other motor systems as well. For example, because in primates there is no one-to-one correspondence between individual fingers and forearm muscles, moving any single digit alone requires coordinating the activity of several muscles that have mechanical actions on many digits (Schieber 1995). Similarly, in systems such as the human jaw in which muscles act across multiple mechanical degrees of freedom, control signals to a large number of muscles must be coordinated appropriately to produce motions in individual degrees of freedom (Gribble et al. 1997; Laboissière et al. 1996).

It was observed in the present studies that the onset of EMG activity in shoulder and elbow muscles followed a proximal to distal temporal ordering-for both single- and multijoint movements, the onset of shoulder muscle activity tended to precede the onset of elbow muscle activity by $\sim 20-50 \mathrm{~ms}$.
These differences in onset timing are presumably not due to neural conduction delays, which would account for $<5 \mathrm{~ms}$ of the time difference. The timing differences observed in the present experiments are consistent with previous reports of muscle activation patterns during multijoint limb movement (Karst and Hasan 1991; Wadman et al. 1980). In addition, McKiernan et al. (1998) report a similar temporal ordering, from proximal to distal muscles, of corticomotoneuronal activation of arm muscles. Similarly, Murphy, Wong, and Kwan (1998) and Scott (1998) report a temporal ordering of the onset of activity in single neurons projecting to proximal and distal muscles. These consistent temporal ordering effects may reflect an organizing principle for upper body movements and may serve in stabilizing the limb.

Additional factors that influence motor adaptation to movement related loads may be noted. Data from recent behavioral studies suggest that sensory feedback plays a critical role in maintaining the effectiveness of the mechanisms that underlie compensation for intersegmental dynamics. Patients who lack proprioceptive input from their limbs fail to accurately reproduce multijoint arm movements in which interaction torques play a major role (Ghez and Sainburg 1995; Sainburg et al. 1993, 1995). When patients were able to view their limbs during movement, however, their performance was improved markedly, although some differences remained relative to neurologically intact control subjects.

Recent neurophysiological data suggest a possible neural basis for the patterns of inter-joint muscle coordination reported here. McKiernan, Marcario, Karrer, and Cheney (1998) report experiments with awake, behaving monkeys in which spike-triggered averaging techniques were used to study the projections of corticomotoneuronal cells to proximal and distal limb muscles. They found that a large majority of the motor cortex cells examined produced postspike effects in two or more muscles, and moreover, nearly half of the cells examined produced postspike effects in both proximal and distal muscles (similar results also were reported for cells in the red nucleus, see Belhaj-Saif et al. 1998; also see Gibson et al. 1985; van Kan et al. 1993). In one case, individual cells were found that produced postspike facilitation in both single-joint shoulder (pectoralis) and elbow (brachialis) muscles. This pattern of projection from motor cortex to arm muscles, if at all general, would provide a mechanism by which compensation for interaction torques may be achieved. For example, the simultaneous activation of pectoralis and brachialis during flexion movements would tend to offset interaction torques at the shoulder due to flexion at the elbow. It should be emphasized, however, that the corticomotoneuronal system only accounts for a relatively small proportion of overall descending neural drive. Other cortical and spinal systems presumably play a significant role in interjoint coordination.

One implication of the findings presented in this paper is that the nervous system makes use of a representation of limb dynamics to carry out the adjustments to control signals that offset forces arising from intersegmental dynamics. Consistent with this finding, a number of researchers have suggested that predictive compensation for movement related loads is based on "internal models" of the motor apparatus (Jordan and Rumelhart 1992; Wolpert et al. 1995). The hypothesis is that these models are instantiated in neural circuitry and, depending on the formulation, are used to predict the kinematic conse- 
quences of motor commands (the "forward model") and/or to determine the motor commands required to generate specific movement trajectories (the "inverse model") (e.g., Atkeson 1989; Kawato et al. 1990; Uno et al. 1989).

Although the present data are consistent with the general notion of internal models, they do not allow one to resolve a separate but related issue-whether the nervous system uses force or position control or some combination. The present finding - the systematic matching of muscle activity to interaction torque-is clearly compatible with models of control (such as inverse dynamics) in which time-varying forces and torques are explicitly computed by the nervous system. On the other hand, the sort of adjustments for forces observed in the present studies also may be cast in terms of equilibrium control models. For example, Flash and Gurevich (1997) present an equilibrium-style control model in which the shape of centrally specified equilibrium trajectories is modified to compensate for external loads introduced by a force field. In this scheme, adjustments to control signals modify equilibrium positions to generate compensatory forces rather than specifying time-varying muscle forces directly. An advantage of casting force control problems in the context of an equilibrium point model is that consistent with empiric findings, both in statics (MussaIvaldi et al. 1985; Shadmehr et al. 1993) and during movement (Won and Hogan 1995), mechanical stability is preserved. Force control models such as inverse dynamics do not necessarily provide similar assurances of stability.

The present results do not permit us to differentiate between inverse dynamics and equilibrium style models of control. In particular, models based on the adjustment of a small number of control parameters (e.g., Gribble et al. 1998; Karniel and Inbar 1997; Loeb et al. 1999) may be able to reproduce many of the phenomena reported both here and in the literature on adaptive load compensation. Many ostensibly "complex" features of kinematics and limb impedance may be predicted using rather simple control signals in which values for a small number of parameters need to be specified (for example, the onset time, rate and duration of an equilibrium shift) (Feldman et al. 1990; Flanagan et al. 1993; Flash 1987; Gribble et al. 1998). It should be noted, however, that even in these cases a mapping is required to determine the adjustments to control signals necessary to produce a given pattern of load compensation.

The authors acknowledge D. Shiller and A. Feldman and thank J. R. Flanagan for helpful comments.

This research was supported by National Institute on Deafness and Other Communication Disorders Grant DC-00594, Natural Sciences and Engineering Research Council, Canada, and Fonds pour la Formation de Chercheurs et l'Aide à la Recherche, Québec.

Address for reprint requests: D. J. Ostry, Dept. of Psychology, McGill University, 1205 Dr. Penfield Ave., Montreal, QC H3A 1B1, Canada.

Received 23 March 1999; accepted in final form 17 June 1999.

\section{REFERENCES}

Almeida, G. L., Hong, D. A., Corcos, D., And Gottlieb, G. L. Organizing principles for voluntary movement: extending single-joint rules. J. Neurophysiol. 74: 1374-1381, 1995.

AtKeson, C. G. Learning arm kinematics and dynamics. Annu. Rev. Neurosci. 12: 157-183, 1989.

Belhaj-Saif, A., Karrer, J. H., And Cheney, P. D. Distribution and characteristics of post-stimulus effects in proximal and distal forelimb muscles from red nucleus in the monkey. J. Neurophysiol. 79: 1777-1789, 1998.
BRown, S. H. AND CoOKE, J. D. Amplitude- and instruction-dependent modulation of movement-related electromyogram activity in humans. J. Physiol. (Lond.) 316: 97-107, 1981.

COOKE, J. D. AND VIRJI-BABUL, N. Reprogramming of muscle activation patterns at the wrist in compensation for elbow reaction torques during planar two-joint arm movements. Exp. Brain Res. 106: 177-180, 1995.

Corcos, D. M., Gottlieb, G. L., And Agarwal, G. C. Organizing principles for single-joint movements. II. A speed-sensitive strategy. J. Neurophysiol. 62: $358-368,1989$.

de Wolf, S., Slijper, H., and Latash, M. L. Anticipatory postural adjustments during self-paced and reaction-time movements. Exp. Brain Res. 121: 7-19, 1998.

Feldman, A. G., Adamovich, S. V., Ostry, D. J., and Flanagan, J. R. The origin of electromyograms-Explanations based on the equilibrium point hypothesis. In: Multiple Muscle Systems: Biomechanics and Movement Organization, edited by J. Winters and S. Woo. New York: Springer-Verlag, 1990, p. 195-213.

Flanagan, J. R., Ostry, D. J., and Feldman, A. G. Control of trajectory modifications in target-directed reaching. J. Mot. Behav. 25: 140-152, 1993.

Flanagan, J. R. AND Wing, A. M. The role of internal models in motion planning and control: evidence from grip force adjustments during movements of hand-held loads. J. Neurosci. 17: 1519-1528, 1997.

FLASH, T. The control of hand equilibrium trajectories in multi-joint arm movements. Biol. Cybern. 57: 257-274, 1987.

Flash, T AND GUREVICH, I. Models of motor adaptation and impedance control in human arm movements. In: Self-Organization, Computational Maps and Motor Control, edited by P. Morasso and V. Sanguineti. Amsterdam: Elsevier North-Holland, 1997, p. 423-481.

GHEZ, C. AND SAINBURG, R. Proprioceptive control of interjoint coordination. Can. J. Physiol. Pharmacol. 73: 273-284, 1995.

Gibson, A. R., Houk, J. C., AND KoHLERMAn, N. J. Magnocellular red nucleus activity during different types of limb movement in the macaque monkey. J. Physiol. (Lond.) 358: 527-549, 1985.

Gomi, H. AND Osu, R. Human Arm Stiffness and Viscosity in Interaction with Environments on a Horizontal Plane. Kanagawa, Japan,: Information Science Research Laboratory, NTT, 1996. (Technical Report ISRL-96-3).

Gottlieb, G. L., Song, Q., Hong, D. A., Almeida, G. L., And Corcos, D. Coordinating movement at two joints: a principle of linear covariance. J. Neurophysiol. 75: 1760-1764, 1996.

Gribble, P., LABoissière, R., AND Ostry, D. Control of human arm and jaw motion: Issues related to musculo-skeletal geometry. In: Self-Organization, Computational Maps and Motor Control, edited by P. Morasso and V. Sanguineti. Amsterdam: Elsevier North-Holland, 1997, vol. 119, p. 483506.

GRIBble, P. L. AND Ostry, D. J. Independent coactivation of shoulder and elbow muscles. Exp. Brain Res. 123: 355-360, 1998.

Gribble, P. L., Ostry, D. J., Sanguineti, V., and Laboissière, R. Are complex control signals required for human arm movement? J. Neurophysiol. 79: 1409-1424, 1998.

Haggard, P., Hutchinson, K., and Stein, J. Patterns of coordinated multijoint movement. Exp. Brain Res. 107: 254-266, 1995.

Hollerbach, J. M. and Flash, T. Dynamic interactions between limb segments during planar arm movement. Biol. Cybern. 44: 67-77, 1982.

JoRdAN, M. I. AND RumelharT, D. E. Forward models: supervised learning with a distal teacher. Cognit. Sci. 16: 307-354, 1992.

KARNIEL, A. AND InBAR, G. F. A model for learning human reaching movements. Biol. Cybern. 77: 173-183, 1997.

KARST, G. M. AND HASAN, Z. Timing and magnitude of electromyographic activity for two-joint arm movements in different directions. J. Neurophysiol. 66: 1594-1604, 1991.

Kawato, M., Maeda, Y., Uno, Y., and Suzuki, R. Trajectory formation of arm movement by cascade neural network model based on minimum torquechange criterion. Biol. Cybern. 62: 275-288, 1990.

Koshland, G. F., Gerilovsky, L., And Hasan, Z. Activity of wrist muscles elicited during imposed or voluntary movements about the elbow joint. $J$. Mot. Behav. 23: 91-100, 1991.

Laboissière, R., Ostry, D. J., and Feldman, A. G. The control of multimuscle systems: human jaw and hyoid movements. Biol. Cybern. 74: 373-384, 1996.

Loeb, G. E., Brown, I. E., And ChEng, E. J. A heirarchical foundation for models of sensorimotor control. Exp. Brain Res. 126: 1-18, 1999. 
McKiernan, B. J., Marcario, J. K., Karrer, J. H., and Cheney, P. D. Corticomotoneuronal postspike effects in shoulder, elbow, wrist, digit and intrinsic hand muscles during a reach and prehension task. J. Neurophysiol. 80: 1961-1980, 1998.

Morasso, P. Spatial control of arm movements. Exp. Brain Res. 42: 223-227, 1981.

Morasso, P. Three dimensional arm trajectories. Biol. Cybern. 48: 187-194, 1983.

Murphy, J. T., Wong, Y. C., And Kwan, H. C. Sequential activation of neurons in primate motor cortex during unrestrained forelimb movement. J. Neurophysiol. 53: 435-445, 1998.

Mussa-Ivaldi, F. A., Hogan, N., And Bizzi, E. Neural, mechanical, and geometric factors subserving arm posture in humans. J. Neurosci. 5: 2732 2743, 1985.

Mustard, B. E. And LeE, R. G. Relationship between emg patterns and kinematic properties for flexion movements at the human wrist. Exp. Brain Res. 66: 247-256, 1987.

Sainburg, R. L., Ghilardi, M. F., Poizner, H., and Ghez, C. Control of limb dynamics in normal subjects and patients without proprioception. J. Neurophysiol. 73: 820-835, 1995.

SAinburg, R. L., Poizner, H., AND GHez, C. Loss of proprioception produces deficits in interjoint coordination. J. Neurophysiol. 70: 2136-2147, 1993.

SCHIEBER, M. H. Muscular production of individuated finger movements: the roles of extrinsic finger muscles. J. Neurosci. 15: 284-297, 1995.

ScotT, S. H. Comparison of onset time and magnitude of activity for proximal arm muscles and motor cortical cells before reaching movements. J. Neurophysiol. 77: 1016-1022, 1998.
Shadmehr, R., Mussa-Ivaldi, F. A., And Bizzi, E. Postural force fields of the human arm and their role in generating multijoint movements. J. Neurosci. 13: 45-62, 1993.

Soechting, J. F. AND LACQUANITI, F. Invariant characteristics of a pointing movement in man. J. Neurosci. 1: 710-720, 1981.

Uno, Y., Kawato, M., AND SuzuKi, R. Formation and control of optimal trajectory in human arm movement. Biol. Cybern. 61: 89-101, 1989.

VAN DER FITS, I. B. AND HADDERS-ALGRA, M. The development of postural response patterns during reaching in healthy infants. Neurosci. Biobehav. Rev. 22: 521-526, 1998.

VAN Kan, P. L., HouK, J. C., AND Gibson, A. R. Output organization of intermediate cerebellum of the monkey. J. Neurophysiol. 69: 57-73, 1993.

VIRJI-BABUL, N. AND COOKE, J. D. Influence of joint interactional effects on the coordination of planar two-joint arm movements. Exp. Brain Res. 103: 451-459, 1995.

WAdman, W. J., van Der Gon, J.J.D., AND DeRKSEn, R. J. Muscle activation patterns for fast goal-directed arm movements. J. Hum. Mov. Stud. 6: 19-37, 1980 .

WiNTER, D. Biomechanics of Human Movement. New York: John Wiley, 1990.

Wolpert, D. M., Ghahramani, Z., And Jordan, M. I. An internal model for sensorimotor integration. Science 269: 1880-1882, 1995.

Won, J. AND HogAN, N. Stability properties of human reaching movements. Exp. Brain Res. 107: 125-136, 1995.

Yamaguchi, K., Riew, K. D., Galatz, L. M., Syme, J. A., And Neviaser, R. J. Biceps activity during shoulder motion: an electromyographic analysis. Clin. Orthop. 336: 122-129, 1997. 